\title{
Varying the light quark mass: Impact on the nuclear force and big bang nucleosynthesis
}

\author{
J. C. Berengut, ${ }^{1}$ E. Epelbaum, ${ }^{2}$ V. V. Flambaum, ${ }^{1}$ C. Hanhart, ${ }^{3,4,5}$ U.-G. Meißner, ${ }^{3,4,5,6,7,8}$ J. Nebreda, ${ }^{9}$ and J. R. Peláez ${ }^{10}$ \\ ${ }^{1}$ School of Physics, University of New South Wales, Sydney, New South Wales 2052, Australia \\ ${ }^{2}$ Institut für Theoretische Physik II, Ruhr-Universität Bochum, D-44780 Bochum, Germany \\ ${ }^{3}$ Institut für Kernphysik and Jülich Center for Hadron Physics, Forschungszentrum Jülich, D-52425 Jülich, Germany \\ ${ }^{4}$ Institute for Advanced Simulation, Forschungszentrum Jülich, D-52425 Jülich, Germany \\ ${ }^{5}$ JARA-Forces And Matter Experiments, Forschungszentrum Jülich, D-52425 Jülich, Germany \\ ${ }^{6}$ Helmholtz-Institut für Strahlen und Kernphysik, Universität Bonn, D-53115 Bonn, Germany \\ ${ }^{7}$ Bethe Center for Theoretical Physics, Universität Bonn, D-53115 Bonn, Germany \\ ${ }^{8}$ JARA-High Performance Computing, Forschungszentrum Jülich, D-52425 Jülich, Germany \\ ${ }^{9}$ Yukawa Institute for Theoretical Physics, Kyoto University, Kyoto 606-8502, Japan \\ ${ }^{10}$ Departamento Física Teórica II, Universidad Complutense, 28040 Madrid, Spain
}

(Received 14 January 2013; published 10 April 2013)

\begin{abstract}
The quark mass dependences of light-element binding energies and nuclear scattering lengths are derived using chiral perturbation theory in combination with nonperturbative methods. In particular, we present new, improved values for the quark mass dependence of meson resonances that enter the nuclear force. A detailed analysis of the theoretical uncertainties arising in this determination is presented. As an application, we derive from a comparison of observed and calculated primordial deuterium and helium abundances a stringent limit on the variation of the light quark mass, $\delta m_{q} / m_{q}=0.02 \pm 0.04$. Inclusion of the neutron lifetime modification, under the assumption of a variation of the Higgs vacuum expectation value that translates into changing quark, electron, and weak gauge boson masses, leads to a stronger limit, $\left|\delta m_{q} / m_{q}\right|<0.009$.
\end{abstract}

DOI: 10.1103/PhysRevD.87.085018

PACS numbers: 26.35.+c, 06.20.Jr, 12.39.Fe, 21.10.Dr

\section{INTRODUCTION}

The Standard Model is widely believed to be a lowenergy manifestation of a more general theory that unifies the four fundamental forces of nature. Several candidate unified theories suggest that spatial and temporal variation of fundamental constants is a possibility, or even a necessity, in an expanding Universe (see, e.g., the reviews in Refs. [1,2]). Studies of big bang nucleosynthesis (BBN) provide a unique probe of the values of fundamental constants in the pre-recombination Universe. A further motivation to consider the response of light nuclei to changes in $m_{q}$, the light quark mass, ${ }^{1}$ is related to anthropic considerations $[3,4]$ that have, e.g., been used in the context of carbon production in hot stars [5-7] in order to understand how much fine tuning is necessary amongst the fundamental parameters of the Standard Model in order to allow life to emerge on Earth. Only now-based on methods as used here-is one really able to study the explicit quark mass dependence of the nuclear forces and nuclear properties, and therefore their impact on, e.g., nuclear abundances, because such issues can only be investigated systematically

\footnotetext{
${ }^{1}$ Throughout most of this paper, we work in the isospin limit $m_{u}=m_{d}$ and only consider the average light quark mass, $m_{q}=$ $\left(m_{u}+m_{d}\right) / 2$; in addition, the quark masses are studied in units of a fixed $\Lambda_{\mathrm{QCD}}$, for only the variation of dimensionless quantities is meaningful. However, in Sec. VII, we address the constraints from neutron $\beta$ decay, which necessarily requires the inclusion of isospin violation by strong and electromagnetic effects.
}

and completely based on chiral effective field theories or lattice simulations (or combinations thereof).

In addition, recent studies of quasar absorption spectra suggest a cosmological gradient in the value of the fine structure constant, $\alpha$, across the Universe [8]. The existence of this spatial variation could be confirmed from complementary astrophysical studies such as big bang nucleosynthesis [9]. If the values of fundamental constants were different in different regions of space at the time of nucleosynthesis, this could be seen in the spatial distribution of primordial deuterium abundances. Note that while $\mathrm{BBN}$ is relatively insensitive to $\alpha$ variation [10], the limits placed on quark mass variation in this work can be related to the variation of $\alpha$ under a range of unification models [11]. Indeed, many of these grand unification theories predict that relative variations in the strong force would be 1 or 2 orders of magnitude larger than those of the electroweak forces (for a simple explanation of this, see, e.g., Ref. [12]). This is also connected to anthropic questions, for if a spatial variation of fundamental constants were to exist, we should not ask how finely tuned the fundamental parameters are, but instead conclude that life emerged in the region of the Universe where the parameters allowed for it.

Relating the observed primordial abundances to the values of fundamental constants at the time of big bang nucleosynthesis requires theoretical models for how the nuclear reaction rates depend on observable quantities such as binding energies and scattering lengths, as well as a model for how those quantities in turn depend on the 
fundamental constants. The former has been dealt with previously in several works (see, e.g., Refs. [10,13-15] and the references therein); in this paper, we provide a response matrix based on the method described in Ref. [13] for some of these quantities which are of importance to the current work. The second part of the problem, relating bulk nuclear quantities to values of fundamental constants, is the focus of this paper.

Most of the previous studies in this context were performed on the basis of model-dependent estimates for quark mass dependences of nuclear properties [16-21]. However, there are two theoretical tools available that allow, in principle, for a model-independent access to quark mass dependences. On the one hand, there is lattice QCD; on the other hand, one has chiral perturbation theory (ChPT). The former is a direct evaluation of QCD in Euclidean space-time, and thus the quark mass dependence is explicit. In the latter case, the operator structure of quark mass terms is fixed by the QCD symmetries; in fact, ChPT is a faithful representation of the spontaneous and explicit chiral symmetry breaking of QCD [22]. The strength parameters [usually called low-energy constants (LECs)] of those operators have to be fixed from other sources. Generally, this is done by comparison with experiment. However, for operators that explicitly involve the quark mass, as is the case here, such a determination is difficult, since in nature the quark masses take definite values. To determine the LECs of such operators, one can fit either to lattice data directly (see, e.g., Ref. [23], where the formalism is outlined for the $\rho$ meson) or from low-energy scattering data when using some unitarization scheme in addition to ChPT (see, e.g., Refs. [24-26]). It should be stressed, however, that in the latter case some model dependence is involved, since the quark mass terms of higher order than what was put in from ChPT are not complete and depend on the scheme used [27]. In some cases, this model dependence can be controlled, to some extent, by a comparison with lattice data.

In Ref. [28], an effective field theory treatment of the impact of quark mass variation on BBN was presented for the first time. In this work, the quark mass dependence of the $N N$ scattering lengths was used as primary input. To constrain these, the results of Ref. [29] were used, since at present the lattice is not sufficiently accurate to provide precise values of these fundamental parameters. Central to the analysis of Ref. [29] was a naturalness assumption for the quark mass corrections to the leading quark-massindependent contact interactions. On the other hand, the same LEC was allowed to vary in a different range in Ref. [30], which led to quite different quark mass variations of the two-nucleon properties. We remark, however, that the considerations in Ref. [29] were consistent with the earlier resonance saturation study of the leading-order and next-to-leading-order contact interactions performed in Ref. [31].
In this work, we study systematically the impact of quark mass variations on two-nucleon observables based on a study of the quark mass dependences of mesons, since those are expected to give the most prominent contributions. In particular, if a strong quark mass dependence of, say, the potential part of the nucleon-nucleon $(N N)$ interaction that comes from $\sigma$ exchange were present-e.g., in Ref. [16], where a striking strange quark mass dependence of the $\sigma$ is conjectured-it might, in the effective field theory where this field is integrated out, lead to an unnaturally enhanced LEC accompanying some contact interaction. An example of such a pattern is apparent in some low-energy constants of dimension 2 that appear in $\pi N$ scattering: when extracted from data in standard ChPT, they appear to be unnaturally large; however, this can be understood phenomenologically by observing that they are mainly dominated by the exchange of $\Delta$ isobars [32]. Consequently, once the $\Delta$ contribution is subtracted, the residual LECs get reduced significantly. Analogously, one should expect that, once all meson-exchange-induced large quark mass effects are treated explicitly, the bulk of the quark mass dependence is included. ${ }^{2}$ Still, such a procedure involves some modeling that induces some systematic uncertainty which is very difficult to specify.

Our main focuses here are the $\sigma$ and the $\rho$ mesons. Both appear as resonances in the two-pion system. The cleanest way to connect their properties to the $N N$ sector is via a dispersion integral of the Omnès type as used, e.g., in Refs. [35,36]. Here, however, we use a method which is technically easier to handle and more transparent, although admittedly of lower theoretical rigor: in Ref. [31] it was shown that the four nucleon operators of the $N N$ potential can be understood quantitatively in terms of the exchange of heavy mesons in the sense of a resonance saturation. In that paper, explicit expressions are presented for this kind of matching. Thus, we here use the following strategy: we determine the quark mass dependence of the light resonances using the methods of Refs. [24-26], which allows us to predict the quark mass dependences of the fournucleon contact terms using the expressions of Ref. [31]. To complete this study, we then quantify the impact of the determined quark mass dependences of the mesons together with that of the nucleon, which is already studied on the lattice, on the $N N$ observables via an explict calculation of scattering lengths. It is important to note that, to our knowledge, no explicit calculations for a dynamic generation of the $\omega$ meson exist. Thus, we assume throughout that its quark mass dependence is the same as that of the $\rho$. Clearly, this should be refined in future studies.

\footnotetext{
${ }^{2}$ In addition, we need to assume that there are no large quark mass dependences coming from sources other than meson exchanges. In this sense, the findings of Ref. [33] are important; for here it is demonstrated that potentially large quark mass dependences induced by $\pi N N$ cuts [34] cancel, once final-state interaction effects are considered explicitly.
} 
The paper is structured as follows: In Sec. II, we derive the quark mass dependence of nucleon and meson masses, which we use to calculate the impact of quark mass variation on the two-nucleon potential in Sec. III. The theoretical uncertainties of our calculation are discussed in Sec. IV. From the two-nucleon observables, we are able to calculate the quark mass dependence of helium nuclei (Sec. V). Finally, in Secs. VI and VII, we calculate the dependence of primordial abundances on nuclear observables and combine this with the results of the previous sections to derive a limit on the variation of the light quark mass at the time of big bang nucleosynthesis.

\section{QUARK MASS DEPENDENCE OF HADRON MASSES}

Here, we study the quark mass dependence of the pertinent hadron masses. The results for each hadron $H$ are most appropriately presented in terms of the dimensionless parameters $K_{H}$ defined via

$$
\frac{\delta M_{H}}{\delta m_{f}}=K_{H}^{f} \frac{M_{H}}{m_{f}},
$$

evaluated at the physical point. Here $m_{f}$ denotes the mass of the quark of flavor $f$, and $M_{H}$ denotes the mass of hadron $H$. In what follows, we will choose $f=q$ for the light quarks (in the isospin limit) and $f=s$ for the strange quark. Note that, although $m_{f}$ by itself is not renormalization-group invariant, the quantity of relevance here—namely $\delta m_{f} / m_{f}$-is, for the same reason that quark mass ratios are well defined.

\section{A. Quark mass dependence of the nucleon mass}

Because of significant advances in lattice QCD, the pion mass dependence of the nucleon, especially, is now known to some precision. E.g., in Ref. [37], the dependence of the nucleon mass on the pion mass squared as calculated by the BMW Collaboration is given. It is straightforward to extract from this the quantity $K_{N}^{q}$ [for the definition, see Eq. (2.1)]. One finds $K_{N}^{q}=0.04$. Note that more recent evaluations from other lattice collaborations give similar results, as nicely reviewed in Ref. [38]. It is also pointed out in that reference that the nucleon mass can be well represented by a linear function of the pion mass in most lattice simulations, which is at odds with the chiral constraints on this observable.

Alternatively, one may determine $K_{N}^{q}$ from the pionnucleon sigma term, $\sigma_{\pi N}$. Reference [39] finds

$$
\sigma_{\pi N}=44.9_{-5.4}^{+1.8} \mathrm{MeV}
$$

On the other hand, in Refs. [40,41] a value

$$
\sigma_{\pi N}=59 \pm 7 \mathrm{MeV}
$$

is found. In what follows, we use the first value, as it is consistent with the classical determination of Ref. [42] based on dispersion relations. A completely reliable upgrade of the value from Ref. [42] can only be obtained based on the recently proposed Roy-Steiner equations for pionnucleon scattering that allow for precise determination of the pion-nucleon scattering amplitude in the physical region as well as inside the Mandelstam triangle $[43,44]$. There is also a large spread of values determined from lattice QCD which encompasses the range of values given above; see Ref. [45] and the recent review by Kronfeld [46].

Using the Feynman-Hellman theorem $\sigma_{f}=$ $m_{f} \partial M_{N} / \partial m_{f}$, one finds straightforwardly $K_{N}^{f}=\sigma_{f} / M_{N}$ and with that, based on the numbers given above,

$$
K_{N}^{q}=0.048_{-0.006}^{+0.002},
$$

consistent with the number quoted above within $2 \sigma$. The values given are significantly lower than those presented in Refs. [17,18] due to the unusually large value of the $\pi N$ sigma term in those works.

\section{B. Quark mass dependence of meson masses}

Because of their nature as (pseudo-)Nambu-Goldstone bosons (NGBs) of the approximate chiral symmetry of the strong interactions, the quark mass dependence of the members of the pseudoscalar octet is given by standard ChPT, which is model independent. At tree level, for the pion, one finds

$$
K_{\pi}^{q}=0.5, \quad K_{\pi}^{s}=0 .
$$

In our calculation, we have included higher-order terms in the light $(u, d)$ quark mass dependence of the pion, using the $S U(2)$ ChPT expansion up to two loops [47]. The strange quark mass dependence of the pion and the quark mass dependence of the kaon and $\eta$ meson (which will be needed later for the calculation of $K_{\rho}^{f}$ and $K_{\sigma}^{f}$ ) have been calculated using $S U$ (3) ChPT to one loop [48]. We remark, however, that these masses are possibly affected by large higher-order corrections - this is an open issue in threeflavor ChPT. For our study, we do not want to enter this discussion, but rather use the next-to-leading-order (NLO) corrections based on the standard scenario for the strange quark condensate. To get at least some feeling for the corresponding theoretical errors, we have estimated the size of the higher-order corrections by treating the ChPT expansions in two different ways: in the first of them, the expansions are written in terms of the physical masses and decay constants of the NGB; in the second, they are written as a function of the tree-level constants $M_{0 \pi}, M_{0 K}$ and $F_{0}$. In our calculations, these tree-level constants are obtained by fitting the ChPT expansions for the masses and decay constants of the NGB to their physical values. Since the difference between the two treatments corresponds to higher orders in the expansion, it serves as an estimate for the systematic error due to the truncation of the ChPT series. 
TABLE I. Estimates for the $K_{R}^{f}$ coefficients and their uncertainties.

\begin{tabular}{lcll}
\hline \hline$K_{\pi}^{q}$ & $0.494_{-0.013}^{+0.009}$ & $K_{\pi}^{s}$ & $0.00 \pm 0.05$ \\
$K_{\rho}^{q}$ & $0.058 \pm 0.002$ & $K_{\rho}^{s}$ & $0.02 \pm 0.04$ \\
$K_{\sigma}^{q}$ & $0.081 \pm 0.007$ & $K_{\sigma}^{s}$ & $0.01 \pm 0.05$ \\
$K_{F_{\pi}}^{q}$ & $0.048 \pm 0.012$ & $K_{F_{\pi}}^{s}$ & $0.00 \pm 0.06$ \\
\hline \hline
\end{tabular}

The values that we provide in the first line of Table I for $K_{\pi}^{f}$ are an average of the results obtained using $S U(2)$ and $S U(3)$, the two methods of truncation mentioned above and different determinations for the ChPT LECs [49-52]. The error is taken so that it covers all the results with their statistical uncertainties, which arise from the errors of the LECs. Following a similar procedure, also in Table I, we provide the $K_{F_{\pi}}^{f}$ values for the pion decay constant $F_{\pi}$.

For the other light resonances the situation is less systematic, since all of them are unstable, and their correct description requires a pole in the complex energy plane that cannot be obtained within the ChPT expansion, which, up to some logarithmic terms, corresponds to an expansion in powers of the energy or the meson masses. By construction, the amplitudes of ChPT are only perturbatively unitary and valid only near threshold. In Ref. [23], a formula to be used in chiral extrapolations for vector meson masses was presented; however, the quality of lattice data was not sufficient to pin down the slope of the quark mass dependence, which is required here. Of course, there are better data now [53-55], so one could refresh the analysis of Ref. [23]. Here, we follow another path, which can also be used to explore the quark mass dependences of the $\rho$ and the $\sigma$. Employing dispersion relations for the inverse $\pi \pi$ scattering amplitude and using ChPT to fix the subtraction constants-where the subtraction points can be chosen in a regime where ChPT is valid-solves both problems and, by generating poles, allows for the study of resonances without a priori assumptions about their existence or nature. This is done in a way consistent with the ChPT expansion without introducing spurious parameters where an uncontrolled quark mass dependence may appear. $^{3}$ This method is called the inverse amplitude method (IAM) [56] and has been used to study both the $\sigma$ and the $\rho$ in an $S U(2)$ one-loop treatment in Ref. [24] and to two loops in Ref. [25]. The $S U(3)$ version of this study can be found in Ref. [26].

Let us note that, within the IAM, all the dependence on the quark masses appears through the NGB masses, which are explicitly present in the amplitudes, both kinematically

\footnotetext{
${ }^{3}$ Clearly, the expansion is controlled only to the order of the chiral expansion used as input. Terms of higher order produced by the formalism are not necessarily correct [27], although at least they will respect unitarity and the correct analytic structure of the amplitude.
}

and in interaction vertices. Thus, we can calculate the $K_{R}^{f}$ parameters for the resonances generated within the IAM by varying the masses of the NGBs, whose dependence on the quark masses was discussed in the previous section, and measuring the corresponding change on the position of the poles.

We have performed this calculation using $S U(2)$ and $S U(3)$ and different sets of LECs obtained from IAM fits [24-26,57]. In each case, we have changed the masses of the NGB using the two different methods for the truncation of the ChPT expansion commented on above. Our estimates for the $K$ factors for $\rho$ and $\sigma$, given in the second and third rows of Table I, are an average of the results combining these different approaches, with errors taken to cover all the results. Let us note that the description of the $\sigma$ depends more strongly on the chiral loops, which are model independent, and much less on the LECs. However, the dependence of the $\rho$ resonance on the quark masses depends strongly on the values of the LECs. For that reason, for the central value of $K_{\rho}^{q}$ we have only used the averaged results of the two best two-loop $S U(2) \mathrm{ChPT}$ fits in Ref. [25], which we consider to be the most reliable, in particular because they were fitted to three sets [53] of lattice calculations of the $\rho$ mass dependence on the pion mass, which were consistent among themselves, ${ }^{4}$ and because the resulting values of the LECs were more consistent with standard determinations and estimates. We refer the reader to Ref. [25] for details. For the strange quark mass dependence, we rely on the existing IAM one-loop $S U(3)$ calculations in Ref. [26], but include the uncertainties as just described above.

It should be stressed that the quark mass dependences we find are significantly smaller than those given in Ref. [16]. In particular, in that reference a value of $K_{\sigma}^{s}=0.54$ is given, compared to our -0.01 (cf. Table I). The origin for this significant discrepancy is the assumption about the quark structure of the $\sigma$ underlying the work of Ref. [16]: the $\sigma$ was assumed to be an $S U(3)$ singlet. In our case, on the other hand, the $\sigma$ emerges from nonperturbative $\pi \pi$ interactions, which give only a very small dependence on the strange quark mass.

As becomes clear from Table I, for all relevant quantities, the variation with respect to the strange quark mass is smaller than the corresponding uncertainty. In addition, some quark mass variations driven by an external scale will lead to a relative change in the strange sector suppressed additionally by a factor $m_{q} / m_{s} \sim 1 / 25$. In what follows, we will therefore only study the effect of a variation of the light quark masses on the $N N$ potential.

\footnotetext{
${ }^{4}$ Let us nevertheless remark that there are other lattice calculations which are not quite compatible with these three because their $\rho$ masses fall systematically either above [54] or below [58] them. The ones falling below are somewhat harder to accommodate within the IAM, as explained in Ref. [25].
} 


\section{IMPACT ON THE VARIATION OF THE TWO-NUCLEON POTENTIAL}

The changes in the meson masses cannot be directly connected to their impact on BBN. The quantity of relevance is the resulting variation of the two-nucleon $(N N)$ interaction, and especially its impact on nuclear binding energies. However, the connection of meson masses to the $N N$ interaction is not clear cut. In Ref. [12], the phenomenological V18 interaction was used as a basis, where the insights of Refs. $[17,18]$ were translated into a variation of the model parameters. Although it provides some understanding of the sensitivity of the $N N$ interaction on the variation of quark mass parameters, such a calculation is neither systematic nor complete. On the other hand, in Ref. [28] an EFT approach is chosen; however, there the input of the quark mass dependence of $N N$ scattering lengths is taken from other sources. Our goal is to improve our understanding of the quark mass dependence of the $N N$ observables, using as input the $K$ factors given in Table I.

As outlined in the Introduction, we do not do the full dispersion theoretical treatment of Refs. [35,36], but rather make a connection to the $N N$ force via the method of resonance saturation: in Ref. [31], explicit expressions are given that allow one to express the values of the $N N$ contact terms in terms of meson masses and coupling constants. Thus, the quark mass dependences given above can be implemented straightforwardly.

\section{A. Quark mass dependence of the pion exchange contributions}

The long-range part of the $N N$ potential up to next-tonext-to-leading order $\left(\mathrm{N}^{2} \mathrm{LO}\right)$ in the chiral expansion is driven by the exchanges of one and two pions. In the exact isospin limit, the one-pion $(1 \pi)$ exchange potential at $\mathrm{N}^{2} \mathrm{LO}$ has the form

$V_{1 \pi}=-\frac{1}{4 F_{\pi}^{2}}\left(g_{A}-2 d_{18} M_{\pi}^{2}\right)^{2} \tau_{1} \cdot \tau_{2} \frac{\left(\vec{\sigma}_{1} \cdot \vec{q}\right)\left(\vec{\sigma}_{2} \cdot \vec{q}\right)}{\vec{q}^{2}+M_{\pi}^{2}}$,

where $\sigma_{i}$ denote the Pauli spin matrices, $\vec{q}=\vec{p}^{\prime}-\vec{p}$ is the nucleon momentum transfer and $\vec{p}\left(\vec{p}^{\prime}\right)$ refers to initial (final) nucleon momenta in the center-of-mass system. Further, $F_{\pi}$ and $g_{A}$ denote the pion decay and the nucleon axial coupling constants, respectively, while $d_{18}$ is a lowenergy constant from $\mathcal{L}_{\pi N}^{(3)}$ that controls the leading contribution to the Goldberger-Treiman discrepancy. Employing the spectral function regularization as detailed in Ref. [59], the nonpolynomial part of the two-pion $(2 \pi)$ exchange potential has the form

$$
\begin{aligned}
V_{2 N}^{(2)}= & -\frac{\tau_{1} \cdot \tau_{2}}{384 \pi^{2} F_{\pi}^{4}} L^{\tilde{\Lambda}}(q)\left(4 M_{\pi}^{2}\left(5 g_{A}^{4}-4 g_{A}^{2}-1\right)+\vec{q}^{2}\left(23 g_{A}^{4}-10 g_{A}^{2}-1\right)+\frac{48 g_{A}^{4} M_{\pi}^{4}}{4 M_{\pi}^{2}+\vec{q}^{2}}\right) \\
& -\frac{3 g_{A}^{4}}{64 \pi^{2} F_{\pi}^{4}} L^{\tilde{\Lambda}}(q)\left(\vec{\sigma}_{1} \cdot \vec{q} \vec{\sigma}_{2} \cdot \vec{q}-\vec{\sigma}_{1} \cdot \vec{\sigma}_{2} \vec{q}^{2}\right) \\
V_{2 N}^{(3)}= & -\frac{3 g_{A}^{2}}{16 \pi F_{\pi}^{4}}\left(2 M_{\pi}^{2}\left(2 c_{1}-c_{3}\right)-c_{3} \vec{q}^{2}\right)\left(2 M_{\pi}^{2}+\vec{q}^{2}\right) A^{\tilde{\Lambda}}(q)-\frac{g_{A}^{2} c_{4}}{32 \pi F_{\pi}^{4}} \tau_{1} \cdot \tau_{2}\left(4 M_{\pi}^{2}+q^{2}\right) A^{\tilde{\Lambda}}(q)\left(\vec{\sigma}_{1} \cdot \vec{q} \vec{\sigma}_{2} \cdot \vec{q}-\vec{q}^{2} \vec{\sigma}_{1} \cdot \vec{\sigma}_{2}\right)
\end{aligned}
$$

with the loop functions $L^{\tilde{\Lambda}}(q)$ and $A^{\tilde{\Lambda}}(q)$ defined as

$$
\begin{gathered}
L^{\tilde{\Lambda}}(q)=\theta\left(\tilde{\Lambda}-2 M_{\pi}\right) \frac{\omega}{2 q} \ln \frac{\tilde{\Lambda}^{2} \omega^{2}+q^{2} s^{2}+2 \tilde{\Lambda} q \omega s}{4 M_{\pi}^{2}\left(\tilde{\Lambda}^{2}+q^{2}\right)}, \quad \omega=\sqrt{4 M_{\pi}^{2}+\vec{q}^{2}}, \quad s=\sqrt{\tilde{\Lambda}^{2}-4 M_{\pi}^{2}}, \\
A^{\tilde{\Lambda}}(q)=\theta\left(\tilde{\Lambda}-2 M_{\pi}\right) \frac{1}{2 q} \arctan \frac{q\left(\tilde{\Lambda}-2 M_{\pi}\right)}{q^{2}+2 \tilde{\Lambda} M_{\pi}} .
\end{gathered}
$$

Here and in what follows, the $c_{i}$ are the LECs from the order- $Q^{2}$ (dimension 2) pion-nucleon Lagrangian, and $\tilde{\Lambda}$ denotes the cutoff in the spectral representation; see Ref. [59]. In addition to the explicit $M_{\pi}$ dependence, at $\mathrm{N}^{2} \mathrm{LO}$ one also needs to take into account the implicit one resulting from the chiral expansion of $g_{A}$ and $F_{\pi}$ in the $1 \pi$ exchange potential in Eq. (3.1). We use the NLO result for the chiral expansion of the pion decay constant, as it is appropriate at the order at which we are working:

$$
F_{\pi}=F\left(1+\frac{M_{\pi}^{2}}{\left(16 \pi^{2} F^{2}\right)} \bar{l}_{4}+\mathcal{O}\left(M_{\pi}^{4}\right)\right),
$$

where $F$ denotes the pion decay constant in the chiral limit. For the LEC $\bar{l}_{4}$, we adopt the value $\bar{l}_{4}=4.3$ from Ref. [60]. Using $F_{\pi}=92.2 \mathrm{MeV}$, this leads to the chiral limit value $F=86.2 \mathrm{MeV}$. Notice that the resulting NLO value for the $K$ factor, $K_{F \pi}^{q}=0.065$, is slightly larger than the one given in Table I. 


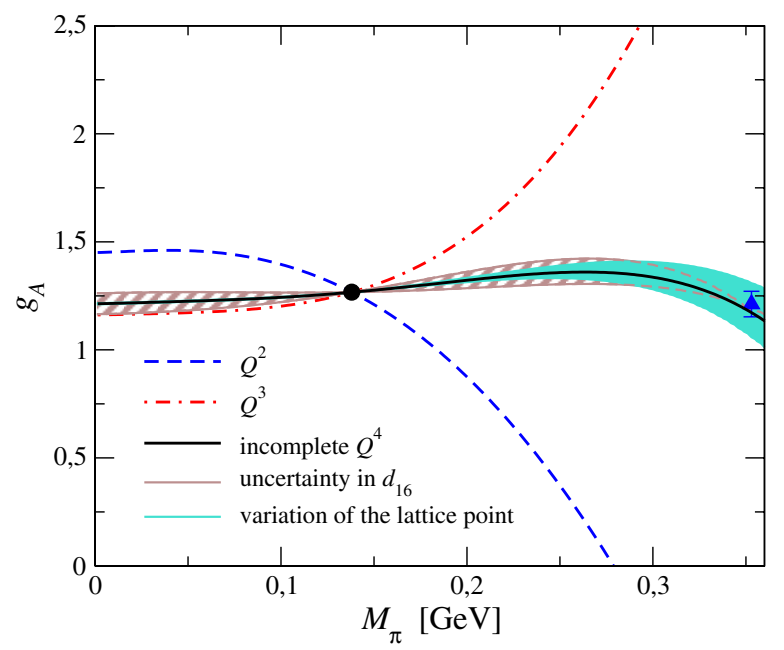

FIG. 1 (color online). Quark mass dependence of $g_{A}$ at various orders in the chiral expansion. Also shown is the lowest mass lattice point from Ref. [63]. The hatched band corresponds to the variation of $\bar{d}_{16}$ in the range $\bar{d}_{16}=-0.91$ to $-2.61 \mathrm{GeV}^{-2}$; see Ref. [61]. The light-shaded band results from a $10 \%$ variation of the lattice point used to fix the LEC of the $M_{\pi}^{4}$ operator in Eq. (3.5).

Contrary to the pion decay constant and the nucleon mass, the chiral expansion for $g_{A}$ is known to converge rather slowly; see Fig. 1. In particular, taking into account the leading [i.e., order $\mathcal{O}\left(M_{\pi}^{2}\right)$ ] correction to its value at the chiral limit and adopting the value for the low-energy constant, $\bar{d}_{16}=-1.76 \mathrm{GeV}^{-2}$, obtained from the reaction $\pi N \rightarrow \pi \pi N$ [61], leads to a very strong quark mass dependence of $g_{A}$ near the physical point. On the other hand, lattice QCD calculations indicate that the behavior of $g_{A}$ with $M_{\pi}$ is rather flat. As discussed in Ref. [62], such a flat behavior of $g_{A}$, consistent with the lowest-mass lattice data point from Ref. [63], corresponding to $M_{\pi}=353 \mathrm{MeV}$, can, in principle, be achieved at the two-loop level. ${ }^{5}$ In order to provide an accurate representation of the quark mass dependence of the $1 \pi$ exchange potential, we use in the present study the complete order- $Q^{3}$ result accompanied by one order- $Q^{4}$ contact term, whose strength is adjusted to reproduce the lowest-mass lattice data from Ref. [62]:

$$
\begin{aligned}
g_{A}= & g_{0}\left[1+\left(\frac{4}{g_{0}} \bar{d}_{16}-\frac{1}{(4 \pi F)^{2}}\left(g_{0}^{2}+\left(2+4 g_{0}^{2}\right) \ln \frac{M_{\pi}}{\bar{M}_{\pi}}\right)\right) M_{\pi}^{2}\right. \\
& \left.+\frac{1}{24 \pi F^{2} m_{0}}\left(3+3 g_{0}^{2}-4 m_{0} c_{3}+8 m_{0} c_{4}\right) M_{\pi}^{3}+\beta M_{\pi}^{4}\right] .
\end{aligned}
$$

Here, $g_{0}, F$ and $m_{0}$ refer to the chiral limit values of the nucleon axial vector coupling, pion decay coupling and nucleon mass, respectively. We use the same values of the

\footnotetext{
${ }^{5} \mathrm{We}$ emphasize, however, that the convergence at such pion masses is problematic, as discussed in detail in Ref. [64].
}

LECs $c_{i}$ as in Ref. [62], ${ }^{6}$ namely $c_{3}=-4.7 \mathrm{GeV}^{-1}, c_{4}=$ $3.5 \mathrm{GeV}^{-1}$. Further, $\bar{M}_{\pi}=138 \mathrm{MeV}$ is the physical value of the pion mass in the isospin limit, while $\beta$ is a linear combination of the order- $Q^{4}$ LECs. We emphasize that the above expression does not correspond to the full order- $Q^{4}$ result, since we do not include the order- $Q^{4}$ chiral logarithms. We have verified numerically that the effect of these logarithms is largely compensated for by the $\beta$ term when the latter is tuned to reproduce the lattice point. Indeed, one observes that the solid line in Fig. 1, corresponding to the pion mass dependence of $g_{A}$ adopted in the present work, is very similar to the more complete calculations of Ref. [62] shown in Fig. 2 of that work. Further, Fig. 1 also shows the uncertainty associated with the variation of $\bar{d}_{16}$ in the range $\bar{d}_{16}=-0.91$ to $-2.61 \mathrm{GeV}^{-2}$ [61] and the variation of the lattice point by $10 \%$.

The value of the nucleon mass in the chiral limit can be obtained from the order- $Q^{3}$ expression

$$
m=m_{0}+4 c_{1} M_{\pi}^{2}+\frac{3 g_{A}^{2}}{32 \pi F_{\pi}^{2}} M_{\pi}^{3}+\mathcal{O}\left(M_{\pi}^{4}\right) .
$$

Using $g_{A}=1.267$ and $c_{1}=-0.81 \mathrm{GeV}^{-1}$ leads to $m_{0}=$ $892 \mathrm{MeV}$. Note that the value of $c_{1}$ used here is consistent with the small sigma term, $\sigma_{\pi N}=45 \mathrm{MeV}$, cf. Eq. (2.2). We further emphasize that the resulting $K$ factor, $K_{N}^{q}=$ 0.042 , is consistent (within the error bars) with the value given in Eq. (2.4).

\section{B. Quark mass dependence of the short-range terms}

The short-range potential in the ${ }^{1} S_{0}$ and ${ }^{3} S_{1}-{ }^{3} D_{1}$ channels up to $\mathrm{N}^{2} \mathrm{LO}$ has the form

$$
\begin{aligned}
& V_{1 S 0}^{\text {short }}=\tilde{C}_{1 S 0}+C_{1 S 0}\left(p^{2}+p^{\prime 2}\right), \\
& V_{3 S 1}^{\text {short }}=\tilde{C}_{3 S 1}+C_{3 S 1}\left(p^{2}+p^{\prime 2}\right), \\
& V_{\epsilon 1}^{\text {short }}=C_{\epsilon 1} p^{2},
\end{aligned}
$$

where $p \equiv|\vec{p}|, p^{\prime} \equiv\left|\vec{p}^{\prime}\right|$ refer to the incoming and outgoing momenta in the center-of-mass system and $\tilde{C}_{i}, C_{i}$ are $M_{\pi}$-dependent coefficients. ${ }^{7}$ The quark mass dependence of these operators can, in principle, be calculated straightforwardly in chiral perturbation theory [29]. The problem is, however, that the coefficients in front of the contact operators $\propto M_{\pi}^{2}$ are unknown. In Ref. [29], the corresponding LECs were estimated by means of naïve dimensional

\footnotetext{
${ }^{6}$ Notice that slightly different values of these LECs are adopted in the chiral $N N$ potential. We have verified that using these different values for $c_{3,4}$ and readjusting the parameter $\beta$ leads to a very similar $M_{\pi}$ dependence of $g_{A}$. The induced difference in the two-nucleon observables is significantly beyond the theoretical uncertainty of our analysis.

${ }^{7}$ Of course, the LECs do not depend on the quark masses. The coefficients used here subsume the coefficients of the LO fournucleon operators plus their first pion mass-dependent corrections that are generated by the same operators times $M_{\pi}^{2}$.
} 

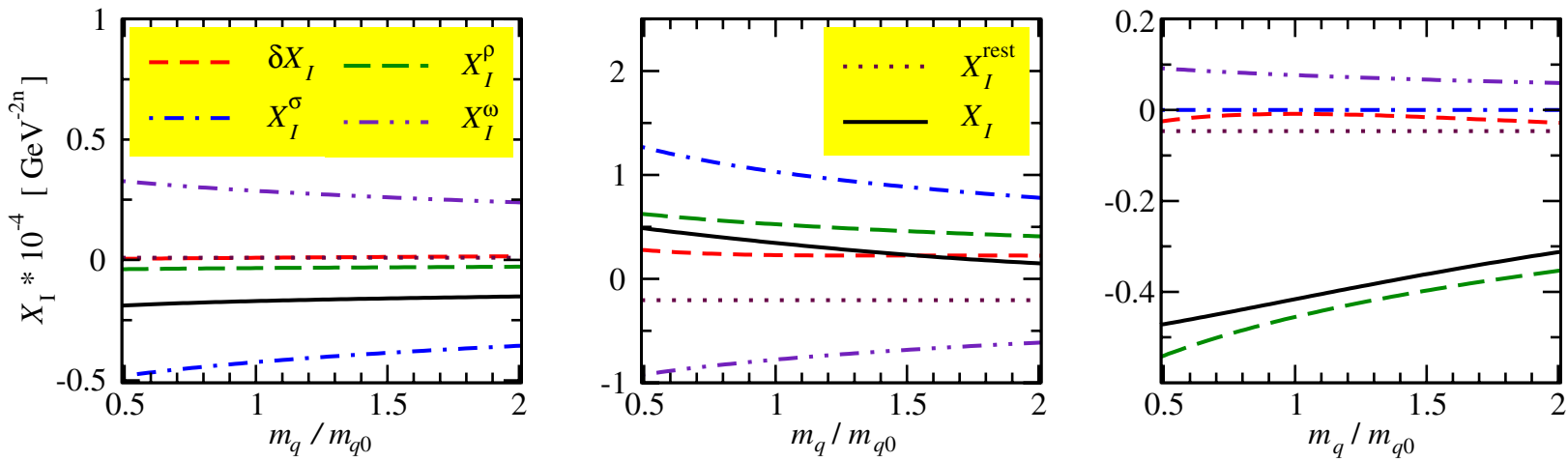

FIG. 2 (color online). Various contributions to the quark mass dependence of the LECs $\tilde{C}_{3 S 1}$ (left panel), $C_{3 S 1}$ (middle panel) and $C_{\epsilon 1}$ (right panel) for fit 1 at $\mathrm{N}^{2} \mathrm{LO}$, as discussed in the text. Here, $m_{q 0}$ denotes the physical value of the light quark mass.

analysis which, however, resulted in a very large theoretical uncertainty for two-nucleon observables. In order to avoid this difficulty, we follow here a completely different approach and refrain from doing an explicit chiral expansion for contact operators. Instead, we make use of resonance saturation of contact interactions [31] and employ the $M_{\pi}$ dependence for the masses of the heavy mesons discussed in Sec. II B.

Resonance saturation for contact $N N$ operators up to $\mathrm{N}^{2} \mathrm{LO}$ is discussed in detail in Ref. [31]. In that work, strongly reduced values of the LECs $c_{i}$ were adopted in order to circumvent a very strong attraction resulting from the isoscalar central two-pion $(2 \pi)$ exchange potential calculated using dimensional regularization. As discussed in Ref. [59], the strong attraction in the $2 \pi$ exchange potential at intermediate and shorter distances can be traced back to the large-mass components in the spectrum which cannot be described reliably within the framework of chiral EFT. In the chiral potentials of Refs. [65,66], the unphysical high-mass components in the two-pion exchange spectrum are cut off by means of the spectral function regularization. We now repeat the analysis of Ref. [31] for the actual version of the chiral $\mathrm{N}^{2} \mathrm{LO}$ potentials and test the validity and accuracy of the resonance saturation hypothesis.

Here and in what follows, we consider the Bonn-B [67] potential as a typical representative of one-boson exchange (OBE) models. Its long-range part is given by $1 \pi$ exchange, whereas shorter-distance physics is expressed as a sum over contributions from the exchange of heavier mesons. For nucleon momentum transfers well below the masses of the exchanged mesons, one can interpret such exchange diagrams in terms of local contact operators with an increasing number of derivatives (momentum insertions). The LECs accompanying the resulting contact interactions are then expressed in terms of the meson masses, the meson-nucleon coupling constants and the corresponding form factors. In order to allow for a meaningful comparison between the chiral and OBE potentials, one needs to properly account for the chiral $2 \pi$ exchange potential, which contributes to the chiral potential but is absent in the OBE models. Here we follow the strategy of Ref. [31] and expand the $2 \pi$ exchange potential of Eq. (3.2) in powers of momenta. The induced contributions to the LECs entering the ${ }^{1} S_{0}$ and ${ }^{3} S_{1}{ }^{3} D_{1}$ partial waves read

$$
\begin{aligned}
& \delta \tilde{C}_{1 S 0}^{(2)}=\frac{\left(-8 g_{A}^{4}+4 g_{A}^{2}+1\right) M_{\pi}^{2} \sqrt{\tilde{\Lambda}^{2}-4 M_{\pi}^{2}}}{24 \pi F_{\pi}^{4} \tilde{\Lambda}}, \\
& \delta C_{1 S 0}^{(2)}=\frac{\sqrt{\tilde{\Lambda}^{2}-4 M_{\pi}^{2}}\left(\left(-88 g_{A}^{4}+17 g_{A}^{2}+2\right) \tilde{\Lambda}^{2}+2\left(8 g_{A}^{4}-4 g_{A}^{2}-1\right) M_{\pi}^{2}\right)}{144 \pi F_{\pi}^{4} \tilde{\Lambda}^{3}}, \\
& \delta \tilde{C}_{3 S 1}^{(2)}=\frac{\left(8 g_{A}^{4}-4 g_{A}^{2}-1\right) M_{\pi}^{2} \sqrt{\tilde{\Lambda}^{2}-4 M_{\pi}^{2}}}{8 \pi F_{\pi}^{4} \tilde{\Lambda}}, \\
& \delta C_{3 S 1}^{(2)}=\frac{\sqrt{\tilde{\Lambda}^{2}-4 M_{\pi}^{2}}\left(\left(40 g_{A}^{4}-17 g_{A}^{2}-2\right) \tilde{\Lambda}^{2}+\left(-16 g_{A}^{4}+8 g_{A}^{2}+2\right) M_{\pi}^{2}\right)}{48 \pi F_{\pi}^{4} \tilde{\Lambda}^{3}}, \\
& \delta C_{\epsilon 1}^{(2)}=-\frac{g_{A}^{4} \sqrt{\tilde{\Lambda}^{2}-4 M_{\pi}^{2}}}{4 \sqrt{2} \pi F_{\pi}^{4} \tilde{\Lambda}}
\end{aligned}
$$


TABLE II. Contributions to the LECs $\tilde{C}_{i}$ and $C_{i}$ induced by the NLO and $\mathrm{N}^{2} \mathrm{LO} 2 \pi$ exchange potential. The $\tilde{C}_{i}$ are in $10^{4} \mathrm{GeV}^{-2}$, and the $C_{i}$ are in $10^{4} \mathrm{GeV}^{-4}$.

\begin{tabular}{lcccc}
\hline \hline LEC & $Q^{2}, \tilde{\Lambda}=500 \mathrm{MeV}$ & $Q^{2}, \tilde{\Lambda}=700 \mathrm{MeV}$ & $Q^{3}, \tilde{\Lambda}=500 \mathrm{MeV}$ & $Q^{3}, \tilde{\Lambda}=700 \mathrm{MeV}$ \\
\hline$\delta \tilde{C}_{1 S 0}$ & -0.004 & -0.005 & -0.004 & -0.005 \\
$\delta C_{1 S 0}$ & -0.534 & -0.592 & -0.365 & -0.500 \\
$\delta \tilde{C}_{3 S 1}$ & 0.013 & 0.014 & -0.004 & -0.005 \\
$\delta C_{3 S 1}$ & 0.594 & 0.663 & -0.365 & -0.500 \\
$\delta C_{\epsilon 1}$ & -0.178 & -0.196 & 0.170 & 0.229 \\
\hline \hline
\end{tabular}

TABLE III. LECs $\tilde{C}_{i}$ and $C_{i}$ from the NLO chiral potential for different cutoff combinations [fits 1 to 5 as defined in Eq. (3.10)], corrected by the induced contributions of the $2 \pi$ exchange potential. Also shown are values resulting from resonance saturation using the Bonn-B model (last column). The $\tilde{C}_{i}$ are in $10^{4} \mathrm{GeV}^{-2}$, and the $C_{i}$ are in $10^{4} \mathrm{GeV}^{-4}$.

\begin{tabular}{lrrrrrr}
\hline \hline & \multicolumn{1}{c}{ Fit 1 } & Fit 2 & Fit 3 & Fit 4 & Fit 5 & Bonn-B \\
\hline$\tilde{C}_{1 S 0}+\delta \tilde{C}_{1 S 0}^{(2)}$ & -0.161 & -0.066 & -0.095 & -0.161 & -0.111 & -0.117 \\
$C_{1 S 0}+\delta C_{1 S 0}^{(2)}$ & 0.974 & 1.574 & 1.457 & 1.008 & 1.386 & 1.276 \\
$\tilde{C}_{3 S 1}+\tilde{C}_{3 S 1}^{(2)}$ & -0.169 & -0.136 & -0.135 & -0.167 & -0.135 & -0.101 \\
$C_{3 S 1}+\delta C_{3 S 1}^{(2)}$ & 0.356 & 0.256 & 0.231 & 0.280 & 0.221 & 0.660 \\
$C_{\epsilon 1}+\delta C_{\epsilon 1}^{(2)}$ & -0.390 & -0.332 & -0.325 & -0.373 & -0.321 & -0.410 \\
\hline \hline
\end{tabular}

at NLO; and

$$
\begin{aligned}
& \delta \tilde{C}_{1 S 0}^{(3)}=\frac{3 g_{A}^{2} M_{\pi}^{3}\left(2 c_{1}-c_{3}\right)\left(2 M_{\pi}-\tilde{\Lambda}\right)}{4 F_{\pi}^{4} \tilde{\Lambda}}, \\
& \delta C_{1 S 0}^{(3)}=-\frac{g_{A}^{2} M_{\pi}\left(2 M_{\pi}-\tilde{\Lambda}\right)\left(\tilde{\Lambda}^{2}\left(-10 c_{1}+11 c_{3}-4 c_{4}\right)+4 M_{\pi}^{2}\left(2 c_{1}-c_{3}\right)+2 \tilde{\Lambda} M_{\pi}\left(2 c_{1}-c_{3}\right)\right)}{16 F_{\pi}^{4} \tilde{\Lambda}^{3}}, \\
& \delta \tilde{C}_{3 S 1}^{(3)}=\frac{3 g_{A}^{2} M_{\pi}^{3}\left(2 c_{1}-c_{3}\right)\left(2 M_{\pi}-\tilde{\Lambda}\right)}{4 F_{\pi}^{4} \tilde{\Lambda}}, \\
& \delta C_{3 S 1}^{(3)}=-\frac{g_{A}^{2} M_{\pi}\left(2 M_{\pi}-\tilde{\Lambda}\right)\left(\tilde{\Lambda}^{2}\left(-10 c_{1}+11 c_{3}-4 c_{4}\right)+4 M_{\pi}^{2}\left(2 c_{1}-c_{3}\right)+2 \tilde{\Lambda} M_{\pi}\left(2 c_{1}-c_{3}\right)\right)}{16 F_{\pi}^{4} \tilde{\Lambda}^{3}}, \\
& \delta C_{\epsilon 1}^{(3)}=\frac{c_{4} g_{A}^{2} M_{\pi}\left(\tilde{\Lambda}-2 M_{\pi}\right)}{2 \sqrt{2} F_{\pi}^{4} \tilde{\Lambda}}
\end{aligned}
$$

at $\mathrm{N}^{2} \mathrm{LO}$. In the limit $\tilde{\Lambda} \rightarrow \infty$, corresponding to dimensional regularization, the above expressions agree with the ones given in Ref. [31]. The size of the $2 \pi$-exchange-induced contributions to the LECs for the two extreme values of the spectral function cutoff $\tilde{\Lambda}$ can be read off Table II.

After these preparations, we are now in the position to test the resonance saturation hypothesis for $\tilde{C}_{i}$ and $C_{i}$. The contributions of the various mesons to the contact operators can be obtained by carrying out partial wave decomposition of the expressions for the boson exchange contributions given in Ref. [31] and expanding the results in powers of momenta. In Tables III and IV, the $2 \pi$-exchange-corrected values of these LECs for five cutoff combinations,

$$
\begin{aligned}
\text { NLO: }\{\Lambda, \tilde{\Lambda}\} & =\{400,500\},\{550,500\},\{550,600\},\{400,700\},\{550,700\}, \\
N^{2} \text { LO: }\{\Lambda, \tilde{\Lambda}\} & =\{450,500\},\{600,500\},\{550,600\},\{450,700\},\{600,700\},
\end{aligned}
$$

are listed together with the values resulting from resonance saturation based on the Bonn-B model. For the considered cutoff combinations, all LECs are reasonably well described in terms of resonance saturation. The agreement is better at $\mathrm{N}^{2} \mathrm{LO}$, most notably for the LEC $C_{3 S 1}$. The estimations based on resonance saturation yield numbers which are typically within $20 \%-30 \%$ of the true values, except for the LO LECs $\tilde{C}_{i}$, which appear to be somewhat 
TABLE IV. LECs $\tilde{C}_{i}$ and $C_{i}$ from the $\mathrm{N}^{2} \mathrm{LO}$ chiral potential for different cutoff combinations [fits 1 to 5 as defined in Eq. (3.10)], corrected by the induced contributions of the $2 \pi$ exchange potential. Also shown are values resulting from resonance saturation using the Bonn-B model (last column). The $\tilde{C}_{i}$ are in $10^{4} \mathrm{GeV}^{-2}$, and the $C_{i}$ are in $10^{4} \mathrm{GeV}^{-4}$.

\begin{tabular}{lrrrrrr}
\hline \hline & \multicolumn{1}{c}{ Fit 1 } & \multicolumn{1}{c}{ Fit 2 } & \multicolumn{1}{c}{ Fit 3 } & \multicolumn{1}{c}{ Fit 4 } & \multicolumn{1}{c}{ Fit 5 } & Bonn-B \\
\hline$\tilde{C}_{1 S 0}+\delta \tilde{C}_{1 S 0}^{(2)}+\delta \tilde{C}_{1 S 0}^{(3)}$ & -0.161 & -0.116 & -0.159 & -0.163 & -0.161 & -0.117 \\
$C_{1 S 0}+\delta C_{1 S 0}^{(2)}+\delta C_{1 S 0}^{(3)}$ & 1.164 & 1.368 & 1.243 & 1.321 & 1.321 & 1.276 \\
$\tilde{C}_{3 S 1}+\delta \tilde{C}_{3 S 1}^{(2)}+\delta \tilde{C}_{3 S 1}^{(3)}$ & -0.162 & -0.127 & -0.137 & -0.164 & -0.130 & -0.101 \\
$C_{3 S 1}+\delta C_{3 S 1}^{(2)}+\delta C_{3 S 1}^{(3)}$ & 0.574 & 0.423 & 0.523 & 0.720 & 0.568 & 0.660 \\
$C_{\epsilon 1}+\delta C_{\epsilon 1}^{(2)}+\delta C_{\epsilon 1}^{(3)}$ & -0.425 & -0.363 & -0.395 & -0.467 & -0.409 & -0.410 \\
\hline \hline
\end{tabular}

more strongly underestimated. For the sake of completeness, we also list in Table $\mathrm{V}$ the contributions from individual meson exchanges in the Bonn-B model; see also Ref. [31].

The observed reasonably good representation of the LECs accompanying the short-range operators in terms of heavy meson exchanges justifies modeling the chiral extrapolations for the corresponding operators in terms of the quark/pion mass dependence of the heavy mesons as discussed in Sec. II B. More precisely, we assume that the resonance saturation hypothesis remains valid at unphysical values of the quark/pion masses; that is,

$$
\begin{aligned}
X_{I}\left(M_{\pi}\right)+\delta X_{I}\left(M_{\pi}\right)= & X_{I}^{\sigma}\left(M_{\pi}\right)+X_{I}^{\rho}\left(M_{\pi}\right) \\
& +X_{I}^{\omega}\left(M_{\pi}\right)+X_{I}^{\mathrm{rest}} .
\end{aligned}
$$

Here $X$ stays for $C, \tilde{C}$ and $I=\{1 S 0,3 S 1, \epsilon 1\}$ and $\delta X_{I}\left(M_{\pi}\right)=\delta X_{I}^{(2)}\left(M_{\pi}\right) \quad$ at $\quad \mathrm{NLO}, \quad$ and $\quad \delta X_{I}\left(M_{\pi}\right)=$ $\delta X_{I}^{(2)}\left(M_{\pi}\right)+\delta X_{I}^{(3)}\left(M_{\pi}\right)$ at $\mathrm{N}^{2} \mathrm{LO}$. For the resonance contributions, we take into account the quark mass dependence of the $\sigma$ - and $\rho$-meson masses as given in Table I and assume $K_{\omega}^{q}=K_{\rho}^{q}$ for the $\omega$ meson $[17,18]$. Neglecting the quark mass dependence of the $\eta$ and $\delta$ mesons is justified by their small contributions to the LECs. The resulting error is expected to be well below the theoretical uncertainty of our analysis. Notice that in $X_{i}^{\sigma, \rho, \omega}$, we also take into account the quark mass dependence of the nucleon mass. The last term on the right-hand side of Eq. (3.11), $X_{i}^{\text {rest }}$, denotes the contributions to the LECs not related to the heavy boson exchanges. The (unknown) $M_{\pi}$ dependence of $X_{I}^{\text {rest }}$ is neglected in the present work. This, of

TABLE V. Contributions of the various boson exchanges to the LECs for the Bonn-B potential and the corresponding sum. The $\tilde{C}_{i}$ are in $10^{4} \mathrm{GeV}^{-2}$, and the $C_{i}$ are in $10^{4} \mathrm{GeV}^{-4}$.

\begin{tabular}{lrrrrrr}
\hline \hline LEC & \multicolumn{1}{c}{$\eta$} & \multicolumn{1}{c}{$\sigma$} & \multicolumn{1}{c}{$\delta$} & \multicolumn{1}{c}{$\omega$} & \multicolumn{1}{c}{$\rho$} & \multicolumn{1}{c}{ Sum } \\
\hline$\tilde{C}_{1 S 0}^{\text {res }}$ & 0.000 & -0.392 & -0.023 & 0.287 & 0.011 & -0.117 \\
$C_{1 S 0}^{\text {res }}$ & 0.033 & 1.513 & 0.036 & -0.560 & 0.254 & 1.276 \\
$\tilde{C}_{3 S 1}^{\text {res }}$ & 0.000 & -0.424 & 0.070 & 0.287 & -0.034 & -0.101 \\
$C_{3 S 1}^{\text {res }}$ & -0.011 & 1.030 & -0.108 & -0.777 & 0.526 & 0.660 \\
$C_{\epsilon 1}^{\text {res }}$ & -0.032 & 0.000 & 0.000 & 0.077 & -0.455 & -0.410 \\
\hline \hline
\end{tabular}

course, only makes sense if $X_{I}^{\text {rest }}$ is small compared to the $X_{I}$; i.e., if the LECs are well described in terms of resonance saturation. This is indeed the case for both the NLO and $\mathrm{N}^{2} \mathrm{LO}$ potentials. As a representative example, we show in Fig. 2 the individual contributions to the quark mass dependence of $\tilde{C}_{3 S 1}, C_{3 S 1}$ and $C_{\epsilon_{1}}$ resulting from Eq. (3.11) for fit 1 at $\mathrm{N}^{2} \mathrm{LO}$. While strong cancellations between the $\sigma$ and $\omega$ contributions are observed for $\tilde{C}_{3 S 1}$ and $C_{3 S 1}$, the LEC $C_{\epsilon 1}$ is largely saturated by the $\rho$ meson. Notice that as a result of the cancellations, there is a sizeable uncertainty in the $m_{q}$ dependence of $C_{3 S 1}$ associated with the nonresonance contribution of the last term in Eq. (3.11).

\section{Quark mass dependence of the $S$-wave $N N$ observables}

Having specified the quark mass dependence of the $N N$ potential, we now turn to the chiral extrapolations of twonucleon $S$-wave observables. We calculate the $N N$ phase shifts and mixing angles by solving the nonrelativistic ${ }^{8}$ Lippmann-Schwinger (LS) equation in the partial wave basis

$$
\begin{aligned}
T_{l^{\prime} l}^{s j}\left(p^{\prime}, p\right)= & V_{l^{\prime} l}^{s j}\left(p^{\prime}, p\right)+\sum_{l^{\prime \prime}} \int_{0}^{\infty} \frac{d p^{\prime \prime} p^{\prime \prime 2}}{(2 \pi)^{3}} V_{l^{\prime} l^{\prime \prime}}^{s j}\left(p^{\prime}, p^{\prime \prime}\right) \\
& \times \frac{m}{p^{2}-p^{\prime \prime 2}+i \eta} T_{l^{\prime \prime} l}^{s j}\left(p^{\prime \prime}, p\right)
\end{aligned}
$$

as $\eta \rightarrow 0^{+}$. The relation between the on-shell $S$ and $T$ matrices is given by

$$
S_{l^{\prime} l}^{s j}(p)=\delta_{l^{\prime} l}-\frac{i}{8 \pi^{2}} p m T_{l^{\prime} l}^{s j}(p)
$$

Thus, the quark/pion mass dependence in the observables emerges from both the nucleon mass entering the $N N$ Green's function and the potential.

Our results for the chiral extrapolation of the deuteron binding energy and the inverse $S$-wave scattering lengths at

\footnotetext{
${ }^{8}$ Relativistic corrections to the two-nucleon Green's function need to be taken into account starting from $\mathrm{N}^{3} \mathrm{LO}$, which is beyond the scope of the present work.
} 


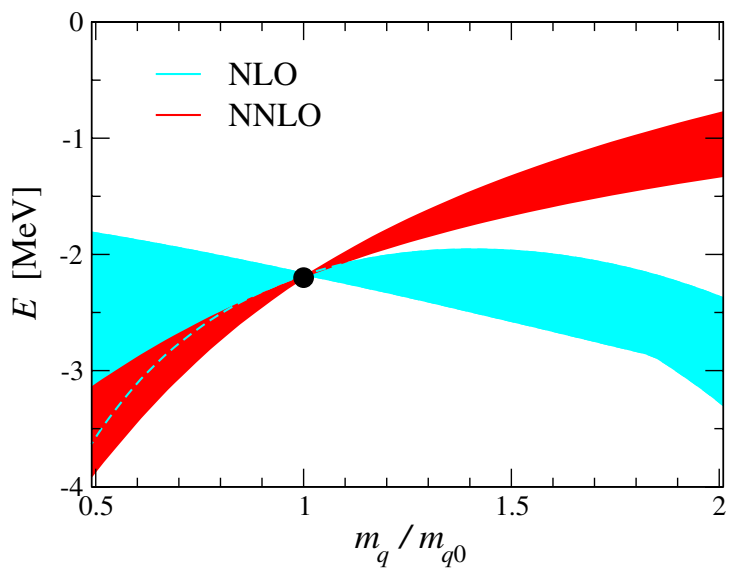

FIG. 3 (color online). Quark mass dependence of the deuteron binding energy at NLO (light-shaded band) and $\mathrm{N}^{2} \mathrm{LO}$ (darkshaded band). The bands correspond to the cutoff variation as discussed in the text. The solid dot shows the deuteron binding energy at the physical quark mass.

NLO and $\mathrm{N}^{2} \mathrm{LO}$ are visualised in Figs. 3 and 4. In these figures, $m_{q 0}$ denotes the physical value of the light quark mass-note that $m_{q} / m_{q 0}$ is, to a very good approximation, equal to $M_{\pi}^{2} / M_{\pi \text {,physical }}^{2}$. In these calculations, we also took into account the implicit quark mass dependence in the twopion exchange potential induced by $g_{A}$ and $F_{\pi}$, which is, strictly speaking, a higher-order effect. We will comment on the size of these higher-order contributions in the next section. Also, as already explained above, we follow here our general strategy and use the most accurate available information regarding the $m_{q}$ dependence of $m$, and especially of $g_{A}$, coming in particular from lattice QCD simulations rather than sticking to the strict chiral expansion at a given order. Note also that within the LS framework, not all contributions to the quark mass dependence are generated, but this effect is well within the error bands discussed later.

We observe the opposite trends in the ${ }^{1} S_{0}$ and ${ }^{3} S_{1}$ channels when changing the value of the quark mass. In particular, the interaction between the nucleons is found to become more attractive in the ${ }^{1} S_{0}$ channel with increasing light quark masses, while it becomes more repulsive in the ${ }^{3} S_{1}$ partial wave. The obtained results do not exclude the possibility of a bound spin singlet state at sufficiently large quark masses. The deuteron is found to remain bound for all values of the quark masses considered. Notice further that our results indicate that the infrared limit cycle proposed in Ref. [68] (see also Ref. [69]) is unlikely to emerge in the range of the quark masses considered in the present analysis. A detailed comparison of our findings with the available calculations will be presented in the next section.

Let us now address the theoretical uncertainty of our calculations. It is comforting to see that the results for the quark mass dependence of the deuteron binding energy and the $S$-wave scattering lengths calculated at NLO and $\mathrm{N}^{2} \mathrm{LO}$ are consistent with each other. The NLO and $\mathrm{N}^{2} \mathrm{LO}$ bands resulting from the cutoff variation as described above overlap, except for large quark masses in the spin triplet channel. This is in line with the observation that the cutoff variation at NLO underestimates the true theoretical uncertainty at this order, since the width of the bands at both NLO and $\mathrm{N}^{2} \mathrm{LO}$ measures the impact of the neglected order- $Q^{4}$ contact interactions. A more complete discussion of the theoretical uncertainties of the calculated $N N$ observables will be given in Sec. IV. Notice further that the chiral extrapolations become rather uncertain for small quark masses, which at first sight might appear counterintuitive. This, however, has to be expected, given that the LECs accompanying contact interactions are fit to experimental data which obviously correspond to the physical quark masses. In addition, one should also keep in mind that we do not rely here on the chiral expansion of the short-range forces, contrary to Refs. [29,34]. Our approach, utilizing resonance saturation and the $K$ factors for heavy meson masses, cannot be expected to yield reliable results at low quark masses, where short-range contributions nonanalytic in quark masses, which are not explicitly taken into account in our calculations, should play an important role.

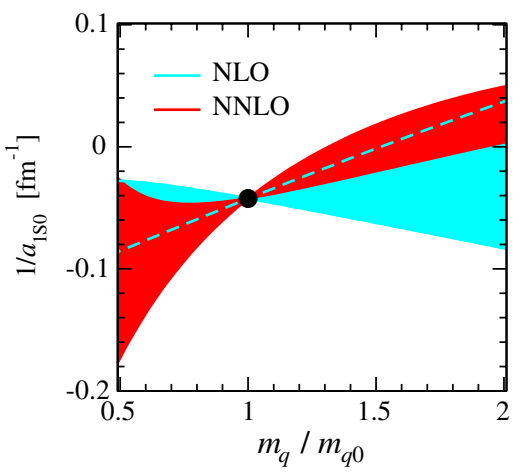

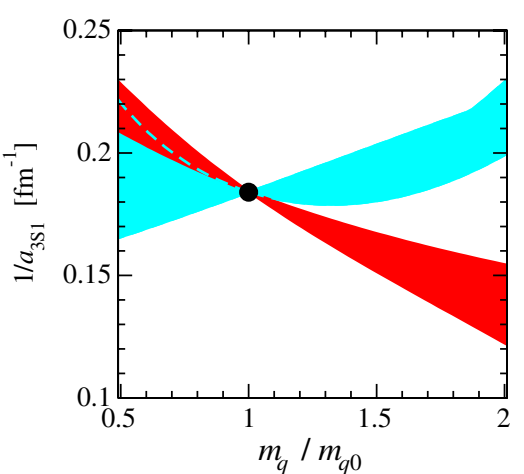

FIG. 4 (color online). Quark mass dependence of the inverse $S$-wave scattering lengths at NLO (light-shaded band) and N ${ }^{2}$ LO (darkshaded band). The bands correspond to the cutoff variation as discussed in the text. The solid dots show the inverse scattering lengths at the physical quark mass. 
TABLE VI. Various contributions to $K_{a, 1 S 0}^{q}, K_{a, 3 S 1}^{q}$ and $K_{\text {deut }}^{q}$. The numbers correspond to the third cutoff combination in Eq. (3.10), while the errors result from the cutoff variations.

\begin{tabular}{|c|c|c|c|c|c|c|}
\hline & \multicolumn{2}{|c|}{$K_{a, 1 S 0}^{q}$} & \multicolumn{2}{|c|}{$K_{a, 3 S 1}^{q}$} & \multicolumn{2}{|c|}{$K_{\text {deut }}^{q}$} \\
\hline & NLO & $\mathrm{N}^{2} \mathrm{LO}$ & NLO & $\mathrm{N}^{2} \mathrm{LO}$ & NLO & $\mathrm{N}^{2} \mathrm{LO}$ \\
\hline$V_{1 \pi}+V_{\mathrm{cont}}^{(0)}$ & $0.2_{-0.6}^{+0.1}$ & $-0.8_{-0.5}^{+0.8}$ & $0.36_{-0.03}^{+0.09}$ & $0.54_{-0.05}^{+0.00}$ & $-0.87_{-0.22}^{+0.06}$ & $-1.28_{-0.0}^{+0.12}$ \\
\hline$+V_{2 \pi}+V_{\mathrm{cont}}^{(2)}$ & $-1.3_{-0.3}^{+2.7}$ & $1.8_{-1.5}^{+1.5}$ & $0.24_{-0.34}^{+0.06}$ & $0.43_{-0.11}^{+0.08}$ & $-0.66_{-0.13}^{+0.80}$ & $-1.11_{-0.19}^{+0.27}$ \\
\hline$+m$ (LS equation) & $-0.6_{-0.2}^{+2.6}$ & $2.3_{-1.5}^{+1.6}$ & $0.13_{-0.33}^{+0.05}$ & $0.32_{-0.10}^{+0.08}$ & $-0.41_{-0.13}^{+0.76}$ & $-0.86_{-0.18}^{+0.24}$ \\
\hline
\end{tabular}

Finally, we list in Table VI the individual contributions of various mechanisms to the dimensionless quantities $K_{\text {deut }}^{q}$ and $K_{a, I}^{q}$, with $I=\{1 S 0,3 S 1\}$, defined as

$$
K_{\text {deut }}^{q}=\frac{\delta E_{\text {deut }} / \delta m_{q}}{E_{\text {deut }} / m_{q}}, \quad K_{a, I}^{q}=\frac{\delta a_{I} / \delta m_{q}}{a_{I} / m_{q}} .
$$

We observe a reasonable convergence pattern in the triplet channel, with the main effect coming from the LO terms in the potential and the contributions due to NLO + NNLO terms and the quark mass dependence of the nucleon mass being considerably smaller. The much larger uncertainty in the singlet channel can be explained by the known feature that the one-pion exchange yields only a minor contribution to the ${ }^{1} S_{0}$ phase shift. The dominant effects emerge from two-pion exchange and shorter-range terms whose quark mass dependence is less constrained than that associated with the longest-range one-pion exchange potential.

\section{DISCUSSION}

We are now in the position to discuss in some detail the theoretical uncertainty of our calculations. Its main sources are due to

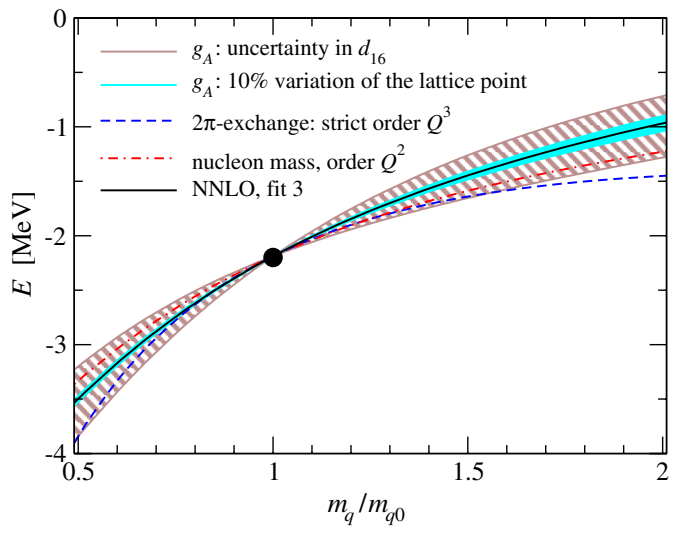

(1) The uncertainty associated with the chiral extrapolation of the nucleon mass $m$ as well as the axial coupling $g_{A}$ and the pion decay constant $F_{\pi}$, which impact the $m_{q}$ dependence of the long-range interactions.

(2) The uncertainty due to truncating the chiral expansion of the potential at $\mathrm{N}^{2} \mathrm{LO}$.

(3) The uncertainty associated with the resonance saturation hypothesis for short-range operators and the employed quark mass dependence of the heavy meson properties.

The chiral dependence of $F_{\pi}$ is well reproduced at the leading-loop order in ChPT, so that the associated uncertainty has a much smaller impact on the two-nucleon observables considered in this work as compared to other sources. On the other hand, the chiral expansion of $g_{A}$ is known to converge slowly. As explained in Sec. III A, we incorporate the order- $Q^{4}$ counterterm and use the lattice QCD result at $M_{\pi}=353 \mathrm{MeV}$ as an input to tune the corresponding LEC. This allows us to obtain a realistic description of the quark mass dependence of $g_{A}$. We check the robustness of this procedure by allowing for a $10 \%$ variation of the lattice point. As visualized in the left panel of Fig. 5, this induces a shift in the binding energies which

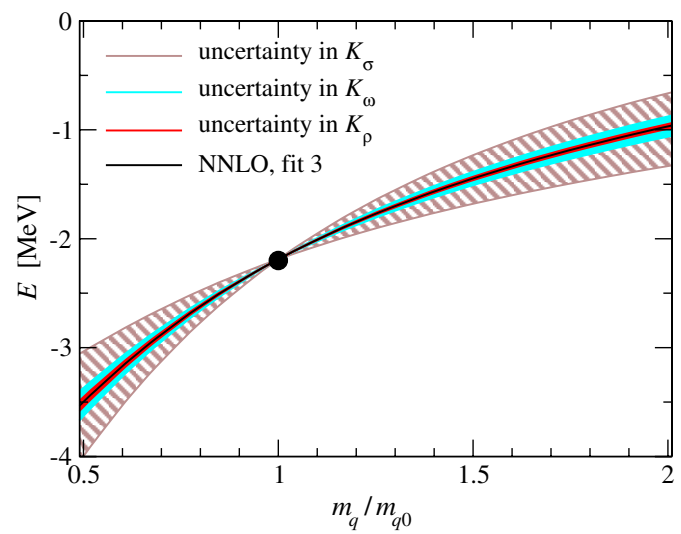

FIG. 5 (color online). Quark mass dependence of the deuteron binding energy at NNLO, fit 3 (solid lines). Left panel: Theoretical uncertainty associated with the quark mass dependence of the long-range interactions and the nucleon mass. The hatched band corresponds to the variation of $\bar{d}_{16}$ in the range $\bar{d}_{16}=-0.91$ to $-2.61 \mathrm{GeV}^{-2}$ (see Ref. [61]). The light shaded band results from a $10 \%$ variation of the lattice point used to fix the order- $Q^{4}$ counterterm in Eq. (3.5). Finally, the dashed line shows the effect of neglecting the quark mass dependence of $g_{A}$ and $F_{\pi}$ in the $2 \pi$ exchange potential, while the dash-dotted line shows the effect of using the order- $Q^{2}$ rather than order- $Q^{3}$ expression [Eq. (3.6)] for the chiral extrapolation of the nucleon mass. Right panel: Theoretical uncertainty induced by the errors in the quark mass dependence of the heavy meson masses according to Table I. 
is considerably smaller than our estimated theoretical uncertainty, cf. Fig. 3. On the other hand, the uncertainty in the determination of the LEC, $\bar{d}_{16}, \bar{d}_{16}=-0.91$ to $-2.61 \mathrm{GeV}^{-2}$ [61], leads to a sizeable spread which is comparable with the one emerging from the cutoff variation. This can be expected, since the value of $\bar{d}_{16}$ influences the shape of the quark mass dependence of $g_{A}$ (largermagnitude values lead to a more flat behavior); see Fig. 1.

The uncertainty due to truncating the chiral expansion for the potential at $\mathrm{N}^{2} \mathrm{LO}$ was already roughly estimated by the cutoff variations; see Fig. 3. As an additional check, we calculated the impact of the $M_{\pi}$ dependence of $g_{A}$ and $F_{\pi}$ in the $2 \pi$ exchange potential, which nominally starts to contribute at $\mathrm{N}^{3} \mathrm{LO}$ (i.e., order $Q^{4}$ ), which is beyond the accuracy of this work. The size of this effect is given by the difference between the solid and dashed lines in the left panel of Fig. 5 and is indeed within the estimated theoretical accuracy at $\mathrm{N}^{2} \mathrm{LO}$. Similarly, it is, strictly speaking, sufficient to use the order- $Q^{2}$ rather than the order- $Q^{3}$ expression for the $M_{\pi}$ dependence of the nucleon mass at $\mathrm{N}^{2} \mathrm{LO}$. The induced difference agrees well with the estimations based on dimensional arguments and is within the accuracy of our calculation; see the dash-dotted line in the left panel of Fig. 5.

Presumably, the most important source of uncertainty is due to the quark mass dependence of the contact interactions. While resonance saturation itself seems, at least in principle, to provide a fairly accurate way to relate the chiral extrapolations of the short-range terms to the properties of heavy mesons which are more easily accessible theoretically, it is difficult to estimate the theoretical uncertainty associated with this procedure. We therefore restrict ourselves to propagating the errors in the $K^{q}$ factors for the heavy meson masses (see Table I) through our analysis. These errors turn out to be strongly magnified due to the large cancellations between the contributions of the $\rho$ and $\omega$ mesons; see Fig. 2. The resulting uncertainty in the deuteron binding energy appears to be comparable to the uncertainty emerging from the cutoff variation and the chiral extrapolations of $g_{A}$; see Fig. 5. We further emphasize that using the linear approximation in terms of the $K$ factors for the quark mass dependence of the heavy mesons is yet another approximation (if one goes sufficiently far away from the physical point). It is, however, irrelevant, as long as one is only interested in the $K$ factors, and can be easily avoided if necessary.

The final results for the $K$ factors of the deuteron binding energy and the corresponding scattering lengths read

$$
\begin{gathered}
K_{a, 1 S 0}^{q}=2.3_{-1.8}^{+1.9}, \quad K_{a, 3 S 1}^{q}=0.32_{-0.18}^{+0.17}, \\
K_{\text {deut }}^{q}=-0.86_{-0.50}^{+0.45},
\end{gathered}
$$

where the numbers are given for the third cutoff combination at $\mathrm{N}^{2} \mathrm{LO}$ and the central values of $K_{\sigma, \rho}^{q}$ listed in Table I. The theoretical uncertainties due to truncating higher-order terms (estimated by the cutoff variation), the uncertainty in $\bar{d}_{16}$, the lattice calculation of $g_{A}$, and the errors in $K_{\sigma, \rho}^{q}$ are added in quadrature.

The results given in Eq. (4.1) can be compared with those of Ref. [29], carried out at NLO in chiral EFT:

$$
\begin{gathered}
K_{a, 1 S 0}^{q}=5 \pm 5, \quad K_{a, 3 S 1}^{q}=1.1 \pm 0.6, \\
K_{\text {deut }}^{q}=-2.8 \pm 1.2,
\end{gathered}
$$

where the numbers are inferred from Figs. 11 and 12 of this work. A more conservative error estimation, taking into account a larger variation in the LEC $\bar{d}_{16}$ and in the quark mass dependence of the lowest-order spin triplet contact interaction, was performed in Ref. [70], leading to

$$
K_{a, 3 S 1}^{q}=1.1 \pm 0.9, \quad K_{\text {deut }}^{q}=-2.9 \pm 1.8 .
$$

As already pointed out before, the major differences between the present analysis and that of Refs. $[29,70]$ are in using a more realistic result for the chiral expansion of $g_{A}$, employing resonance saturation to describe the quark mass dependence of contact interactions and extending the calculations to $\mathrm{N}^{2} \mathrm{LO}$. These improvements have allowed us to determine the values for the $K$ factors with higher accuracy.

The results for both ${ }^{1} S_{0}$ and ${ }^{3} S_{1}$ channels given above are consistent with the chiral EFT values calculated in Ref. [71] using the Kaplan-Savage-Wise approach:

$$
\begin{gathered}
K_{a, 1 S 0}^{q}=2.4 \pm 3.0, \quad K_{a, 3 S 1}^{q}=3.0 \pm 3.5, \\
K_{\text {deut }}^{q}=-7 \pm 6,
\end{gathered}
$$

where the numbers correspond to Figs. 1, 2, and 4 $(\eta=1 / 5)$ of that work.

More recently, attempts have been made to combine chiral EFT with lattice QCD calculations. In particular, the NPLQCD Collaboration has determined the regions for the $S$-wave scattering lengths consistent with their lattice data, $a_{1 S 0}=0.63 \pm 0.50(5-10) \mathrm{fm}$ and $a_{3 S 1}=$ $0.63 \pm 0.74 \quad(5-9) \quad \mathrm{fm}$ obtained at $M_{\pi}=353.7 \pm$ $2.1 \mathrm{MeV}$ [72]. Assuming the validity of the employed chiral EFT frameworks in the considered range of pion masses, they determined the following constraints for the allowed regions of the $K$ factors (the numbers below are extracted from Figs. 3, 4 of Ref. [72]):

$$
K_{a, 1 S 0}^{q} \lesssim-4 \cup K_{a, 1 S 0}^{q} \gtrsim 2
$$

based on the Weinberg approach, and

$$
K_{a, 1 S 0}^{q} \gtrsim 6, \quad-5 \lesssim K_{a, 3 S 1}^{q} \lessgtr-0.2 \cup 0.3 \lesssim K_{a, 3 S 1}^{q} \lesssim 9
$$

using the chiral EFT formulation of Ref. [73]. For a more recent extrapolation of the NPLQCD numbers to physical quark masses, see Ref. [74]. Very recently, a similar analysis has been carried out using the framework of chiral EFT with dibaryon fields [33], yielding $K_{a, 3 S 1}^{q} \sim-0.4$ (see Fig. 9 of that work), and a positive value for $K_{a, 1 S 0}^{q}$ (which 
we were unable to infer from that figure). While this result for $K_{a, 3 S 1}^{q}$ disagrees with our findings, it is difficult to draw conclusions, since Ref. [33] does not provide an estimate of the theoretical uncertainty associated with using the lattice QCD results at large values of the pion mass as input in the calculations. The same comment also applies to the results of Ref. [72] given in Eqs. (4.5) and (4.6), as well as the results of Ref. [74].

Last but not least, it is comforting to see that our results are in good agreement with those of Ref. [12], where the values $K_{\text {deut }}^{q}=-0.75,-0.84$ and -1.39 are reported for three different models of the two-nucleon potentials. Even more important, however, is that we are able to carefully estimate the theoretical uncertainty for this quantity.

\section{CONSEQUENCES FOR HEAVIER NUCLEI}

So far, we focused on the two-nucleon system; however, the quark mass dependences of ${ }^{3} \mathrm{He}$ and ${ }^{4} \mathrm{He}$ are also relevant for BBN. In order to estimate the impact of the quark mass dependences of the deuteron binding energy and the ${ }^{1} S_{0}$ scattering length on BBN, we here use the methods of Ref. [28] - actually, what we have provided in the previous sections is an update of the input used in Ref. [28], which basically came from Ref. [29].

The quark mass dependences of the binding energies of ${ }^{3} \mathrm{He}$ and ${ }^{4} \mathrm{He}$ can be calculated from

$$
K_{A_{\mathrm{He}}}^{q}=K_{a, 1 S 0}^{q} K_{A_{\mathrm{He}}}^{a, 1 S 0}+K_{\mathrm{deut}}^{q} K_{A_{\mathrm{He}}}^{\text {deut }},
$$

where $K_{{ }^{A} \mathrm{He}}^{x}=x / E_{{ }^{A} \mathrm{He}}\left(\delta E_{{ }^{A} \mathrm{He}} / \delta x\right)$ was defined in analogy to the quantities given above. In Ref. [28], the coefficients were calculated from pionless EFT. They read, including the uncertainties quoted in Ref. [28], as follows:

$K_{{ }^{3} \mathrm{He}}^{a, 1 S 0}=0.12 \pm 0.01, \quad K_{{ }^{\mathrm{He}}}^{\text {deut }}=1.41 \pm 0.01 ;$

$K_{{ }^{4} \mathrm{He}}^{a, 1 S 0}=0.037 \pm 0.011, \quad K_{{ }^{4} \mathrm{He}}^{\text {deut }}=0.74 \pm 0.22$.

From this, using the uncertainties for the $K$ factors as given in Eq. (4.1), we get

$$
K_{{ }^{3} \mathrm{He}}^{q}=-0.94 \pm 0.75, \quad K^{q} \mathrm{He}=-0.55 \pm 0.42,
$$

where the uncertainties of Ref. [28] and those quoted in Eq. (4.1) were added in quadrature. Note that there has been a recent lattice study of the deuteron, ${ }^{3} \mathrm{He}$ and ${ }^{4} \mathrm{He}$ at a pion mass of $510 \mathrm{MeV}$ and various lattice sizes [75]. As this pion mass is rather large, we refrain from trying to extract the corresponding $K$ factors from that work. In the future, when such studies become available at lower values of $M_{\pi}$, they will provide valuable constraints on the quark mass dependence of nuclear binding energies. Note further that the $K$ factor for ${ }^{4} \mathrm{He}$ is consistent for the central value obtained from a recent nuclear lattice calculation using the same scattering lengths and $K$ factors, $K_{{ }^{4} \mathrm{He}}^{q}=-0.32$ [7].

\section{IMPACT ON BBN}

In Table VII, we present our calculated BBN response matrix. The quantities presented are the linear dependences of calculated primordial abundances $Y_{a}$ to small variations of nuclear binding energies and scattering lengths $X$, $\partial \ln Y_{a} / \partial \ln X$. They were obtained using the methods and codes (modified from the publicly available Kawano code [76]) described in Ref. [13]. Updated reaction rates are taken from Refs. [77-80]; see Ref. [13] for details. Unlike in previous studies, we have separated the effect of $B_{\text {deut }}$ from the singlet scattering length $a_{s}$. The scattering length affects the rate of the reaction $n(p, d) \gamma$ via the equation (see, e.g., Ref. [81])

$$
\langle\sigma v\rangle \sim\left(B_{\text {deut }}\right)^{5 / 2} / \epsilon_{v},
$$

where $\epsilon_{v}$ is the position of the singlet virtual level. The baryon-to-photon ratio $\eta=6.19(15) \times 10^{-10}$ is taken from the latest WMAP7 data [82].

Final sensitivities of primordial abundances $Y_{a}$ to the variation of $m_{q}$ are obtained by combining the results in Table VII with Eqs. (4.1) and (5.3) using

$$
\frac{\delta \ln Y_{a}}{\delta \ln m_{q}}=\sum_{X_{i}} \frac{\partial \ln Y_{a}}{\partial \ln X_{i}} K_{X_{i}}^{q} .
$$

Error estimates must be performed carefully, since the $K_{X_{i}}^{q}$ are derived from the same sources via Eq. (5.1). We have taken the correlations in errors into account when deriving our final sensitivities. The uncertainties of Eq. (5.2) are also correlated to some extent, but since they are small anyway, we may neglect this correlation. The final sensitivities of primordial abundances to quark mass variation are given in Table VIII, along with final values of quark mass variation at the time of big bang nucleosynthesis extracted from the comparison of observed and calculated primordial abundances.

We see from Table VIII that the deuterium and ${ }^{4} \mathrm{He}$ data give consistent limits on $\delta m_{q} / m_{q}$. Taking a weighted

TABLE VII. BBN response matrix $\partial \ln Y_{a} / \partial \ln X$ at the baryon-to-photon ratio $\eta=6.19 \times 10^{-10}$. The $Y_{a}$ values are the number ratios of primordial isotope abundances to hydrogen, except for ${ }^{4} \mathrm{He}$, which is the mass ratio ${ }^{4} \mathrm{He} / \mathrm{H}$.

\begin{tabular}{lrrrcc}
\hline \hline$X$ & \multicolumn{1}{c}{$d$} & \multicolumn{1}{c}{${ }^{3} \mathrm{He}$} & ${ }^{4} \mathrm{He}$ & ${ }^{6} \mathrm{Li}$ & ${ }^{7} \mathrm{Li}$ \\
\hline$a_{s}$ & -0.39 & 0.17 & 0.01 & -0.38 & 2.64 \\
$B_{\text {deut }}$ & -2.91 & -2.08 & 0.67 & -6.57 & 9.44 \\
$B_{\text {trit }}$ & -0.27 & -2.36 & 0.01 & -0.26 & -3.84 \\
$B^{3} \mathrm{He}$ & -2.38 & 3.85 & 0.01 & -5.72 & -8.27 \\
$B^{{ }^{4}} \mathrm{He}$ & -0.03 & -0.84 & 0.00 & -69.8 & -57.4 \\
$B^{6} \mathrm{Li}$ & 0.00 & 0.00 & 0.00 & 78.9 & 0.00 \\
$B^{B}{ }^{7} \mathrm{Li}$ & 0.03 & 0.01 & 0.00 & 0.02 & -25.1 \\
$B^{7}{ }^{\mathrm{Be}}$ & 0.00 & 0.00 & 0.00 & 0.00 & 99.1 \\
$\tau$ & 0.41 & 0.14 & 0.72 & 1.36 & 0.43 \\
\hline \hline
\end{tabular}


TABLE VIII. Extracted values of quark mass variation, $\delta m_{q} / m_{q}$, during BBN from the comparison of observed and calculated primordial abundances, $Y_{a}$. Observed values are taken from the Particle Data Group review [83], and calculated values are taken from Ref. [84].

\begin{tabular}{lcccr}
\hline \hline$Y_{a}$ & Observed & Calculated & $\delta \ln Y_{a} / \delta \ln m_{q}$ & $\delta m_{q} / m_{q}$ \\
\hline$[$ deut $/ \mathrm{H}]$ & $2.82(21) \times 10^{-5}$ & $2.49(17) \times 10^{-5}$ & $3.9(3.4)$ & $0.034(42)$ \\
${ }^{4} \mathrm{He}\left(Y_{p}\right)$ & $0.249(9)$ & $0.2486(2)$ & $-0.56(34)$ & $-0.003(65)$ \\
\hline \hline
\end{tabular}

average of the two results gives $\delta m_{q} / m_{q}=0.02 \pm 0.04$, our final result.

\section{EFFECT OF THE NEUTRON LIFETIME}

Our limit $\delta m_{q} / m_{q}=0.02 \pm 0.04$ at first appears much weaker than the limit derived by Bedaque et al. [28], who obtained $-1 \% \lesssim \delta m_{q} / m_{q} \lesssim 0.7 \%$, although our input for the two-nucleon parameters is more accurate. The origin of the difference is that, contrary to this work as well as previous works [10, 12, 13], in Ref. [28], a quark mass dependence of the neutron lifetime, $\tau$, was included. Since essentially all free neutrons at the onset of BBN end up in ${ }^{4} \mathrm{He}$ nuclei, and changing $\tau$ changes the neutron-to-proton number ratio at $\mathrm{BBN}$, the ${ }^{4} \mathrm{He}$ abundance is quite sensitive to the neutron decay rate. Therefore, it is worthwhile to examine the assumptions made in Ref. [28] in more detail.

Neutron beta decay becomes possible as a consequence of a nonvanishing proton-neutron mass difference, which is nonzero due to an apparent violation of the isospin symmetry in the Standard Model caused by $m_{u} \neq m_{d}$ and electromagnetic effects driven by different quark charges. One finds

$$
\Delta m_{N}=m_{n}-m_{p}=\Delta m_{N}^{\mathrm{str}}+\Delta m_{N}^{\mathrm{em}}=2 \mathrm{MeV}-0.7 \mathrm{MeV},
$$

with an uncertainty of $0.3 \mathrm{MeV}$ for the individual contributions [85]. ${ }^{9}$ Thus, in order to quantify how $\Delta m_{N}$, and thus $\tau$, changes with the quark masses, an assumption has to be made on how the other Standard Model parameters change - as we will see, of particular importance is the change of the electron mass relative to the quark masses.

The neutron width $\Gamma \sim 1 / \tau$ can be written as

$$
\Gamma=\frac{\left(G_{F} \cos \theta_{C}\right)^{2}}{2 \pi^{3}} m_{e}^{5}\left(1+3 g_{A}^{2}\right) f\left(\frac{\Delta m_{N}}{m_{e}}\right),
$$

where $G_{F}$ is the Fermi constant, $\theta_{C}$ is the Cabibbo angle, $m_{e}$ is the electron mass, and $g_{A}$ is the nucleon axial decay constant. The function $f\left(\Delta m_{N} / m_{e}\right)$ describes the phase space and Coulomb attraction; an explicit form is presented in Ref. [28].

In order to proceed, Ref. [28] made the assumption that when $m_{q}$ changes, $m_{u} / m_{d}$, as well as all other Standard

\footnotetext{
${ }^{9}$ The more recent extraction of Ref. [86] finds $\Delta m_{N}^{\mathrm{em}}=-1.3 \pm 0.5 \mathrm{MeV}$ - consistent within uncertainties.
}

Model parameters, stays constant. This clearly introduces some model dependence. Based on this assumption, one gets a very strong sensitivity of $\Gamma$ to $m_{q}$ via the factor $f$ :

$$
\begin{aligned}
\frac{\delta \ln \Gamma}{\delta \ln m_{q}} & \simeq \frac{\delta \ln f\left(\Delta m_{N} / m_{e}\right)}{\delta \ln m_{q}}=\left.\frac{f^{\prime}}{f}\right|_{\Delta m_{N} / m_{e}}\left(\frac{\delta\left(\Delta m_{N} / m_{e}\right)}{\delta \ln m_{q}}\right) \\
& =10.4 \pm 1.5
\end{aligned}
$$

where $f^{\prime} / f$ is numerically determined to be 2.57 at the physical value of $\Delta m_{N} / m_{e}$. Thus, the large sensitivity to the variation of the quark mass, found in Ref. [28], comes directly from model-dependent assumptions, which allow one to write

$$
\delta\left(\Delta m_{N} / m_{e}\right) / \delta \ln m_{q}=\Delta m_{N}^{\mathrm{str}} / m_{e}=4 .
$$

One the other hand, had one assumed that $m_{u}-m_{d}$ is independent of the quark mass, the value found for $\delta \ln \Gamma / \delta \ln m_{q}$ would be significantly smaller.

In general, how possible changes of fundamental parameters are interrelated depends on the model assumed for the physics beyond the Standard Model. In fact, the relation $m_{u} / m_{d}=$ constant emerges naturally from a scenario where all elementary particle masses are proportional to a varying Higgs vacuum expectation value (VEV), $v$ (relative to $\Lambda_{\mathrm{QCD}}$ ), but the gauge and Yukawa couplings are independent of it. However, in this case $m_{e}$ and the mass of the weak gauge boson, $m_{W}$, are also proportional to $v$. One finds, therefore, that $\Delta m_{N}^{\text {str }} / m_{e}$ has no dependence on $v$, and the sensitivity of $f$ comes from the variation in $m_{e}$ relative to the electromagnetic component of $\Delta m_{N}$ :

$$
\frac{\delta \ln f\left(\Delta m_{N} / m_{e}\right)}{\delta \ln v}=\left.\frac{f^{\prime}}{f}\right|_{\Delta m_{N} / m_{e}}\left(\frac{-\Delta m_{N}^{\mathrm{em}}}{m_{e}}\right)=3.6 \pm 1.5 .
$$

Under our assumption that $\delta \ln m_{W}=\delta \ln m_{e}=$ $\delta \ln m_{q}=\delta \ln v$ (i.e., all masses vary with the Higgs VEV), and noting that $G_{F} \sim 1 / m_{W}^{2}$, from Eq. (7.2), we obtain

$$
\begin{aligned}
\frac{\delta \ln \Gamma}{\delta \ln v} & =1+\frac{3 g_{A}^{2}}{1+3 g_{A}^{2}} \frac{\delta \ln g_{A}^{2}}{\delta \ln v}+\frac{\delta \ln f\left(\Delta m_{N} / m_{e}\right)}{\delta \ln v} \\
& =4.8 \pm 1.5
\end{aligned}
$$

where we used the quark mass dependence of $g_{A}^{2}$ discussed in Sec. III A, which gives 


$$
\frac{\delta \ln g_{A}^{2}}{\delta \ln v}=0.2 \pm 0.1
$$

The final sensitivity to neutron decay rate, $\delta \ln \tau / \delta \ln v=$ -4.8 , is reduced by around a factor of 2 compared with Ref. [28], but it is still very large even within our model. Note that $\delta \ln \tau / \delta \ln v=-4.9$ may also be obtained using Table IV of Dent et al. [10] under the same assumptions, and a value of -4.8 can be extracted from Eq. (7) of Ref. [87]. Multiplying $\delta \ln \tau / \delta \ln v$ by the sensitivity coefficients $\delta \ln Y_{a} / \delta \ln \tau$ presented in Table VII and adding the binding energy and scattering length sensitivities presented in Table VIII (using our assumption $\delta \ln m_{q}=\delta \ln v$ ), we obtain

$$
\frac{\delta \ln Y_{\text {deut }}}{\delta \ln v}=1.9(3.4), \quad \frac{\delta \ln Y_{{ }^{4}} \mathrm{He}}{\delta \ln v}=-4.0(0.3) .
$$

In this model, the $\tau$ sensitivity reduces the deuterium sensitivity to $v$ by a factor of 2 and entirely dominates the ${ }^{4} \mathrm{He}$ sensitivity. The final limits on Higgs VEV variation from deuterium and helium abundances are $\delta v / v=$ $0.07 \pm 0.13$ and $\delta v / v=0.000 \pm 0.009$, respectively. However, we should include here the disclaimer that, although we included the effects of isospin violation in the evaluation of the neutron beta decay, all few-nucleon calculations were done imposing isospin symmetry. One expects these effects to be small compared to that from neutron beta decay; still, for consistency, such effects will have to be included in the future.

Therefore, imposing the model that all masses scale with $v$ and including the variation of $\tau$; as expected, the results are entirely dominated by the ${ }^{4} \mathrm{He}$ data, and the limits are rather tight, $|\delta v / v|<0.9 \%$. Finally, we note that this result does not include all possible mass variations: for example, in Ref. [10], the dependences of primordial abundances on $m_{e}$ are calculated assuming constant $\tau$ (that is, the $m_{e}$ dependence that does not come via $\tau$ variation). In comparison with the dependence from $\tau$, these are rather small, $\delta \ln Y_{a} / \delta \ln m_{e}=-0.16$ and -0.71 for deuterium and ${ }^{4} \mathrm{He}$, respectively. Including this effect would marginally tighten our limits on $\delta v / v$.

\section{SUMMARY}

We have presented a systematic study of the impact of quark mass variations on properties of the two-nucleon system based on chiral perturbation theory combined with nonperturbative techniques. Since the approach is based on an effective field theory, a reliable error estimate becomes feasible - a clear advantage compared to purely phenomenological studies as reported, e.g., in Refs. [16-21]. We include the uncertainties from the quark mass dependence of meson masses as well as those from the treatment of the $N N$ interaction derived from chiral perturabtion theory. Here the heavy (heavier than the pion) meson masses enter into the expressions for four-nucleon contact terms with strengths fixed from a resonance saturation hypothesis - the uncertainty induced by this method is not included. However, support for this procedure comes from the apparent quantitative success of the resonance saturation for four-nucleon contact interactions. In particular, we find

$$
\begin{gathered}
K_{a, 1 S 0}^{q}=2.3_{-1.8}^{+1.9}, \quad K_{a, 3 S 1}^{q}=0.32_{-0.18}^{+0.17}, \\
K_{\text {deut }}^{q}=-0.86_{-0.50}^{+0.45},
\end{gathered}
$$

where the uncertainties are significantly reduced compared to the numbers derived from earlier studies within effective field theory $[29,30]$. The numbers for the $K$ factors presented in this work are the necessary input for studies that address the quark mass dependence of nuclear properties. These studies allow one to quantify how much fine tuning is necessary amongst the Standard Model parameters to allow life to develop-for the most recent developments in this respect, see Ref. [7].

From the given $K$ factors, we derived the quark mass dependence of helium nuclei using the techniques of Ref. [28]. Additionally, we have presented a new response matrix of calculated primordial abundances to variations in nuclear binding energies and the scattering length of the important two-nucleon ${ }^{1} S_{0}$ channel. Combining these, we have derived a stringent limit on the quark mass variation at the time of big bang nucleosynthesis, $\delta m_{q} / m_{q}=$ $0.02 \pm 0.04$.

In previous phenomenological studies, the bounds derived from the deuteron and ${ }^{4} \mathrm{He}$ abundances are, e.g., $0.009 \pm 0.19$ and $-0.005 \pm 0.038$, respectively, from Ref. [12]; and $-0.002 \pm 0.037$ and $0.012 \pm 0.011$, respectively, from Ref. [13]. The uncertainties are significantly smaller compared to ours, since in these works no attempt was made to quanitify the theoretical uncertainty-in Ref. [12], it is stated that the uncertainty is expected to be of the order of a factor of 2 . In this sense, although the uncertainty of our work seems larger, still the bound derived is more robust, since a careful uncertainty estimate was done.

In Ref. [28], a range $-1 \% \leq \delta m_{q} / m_{q} \leq 0.7 \%$ is quoted for the quark mass variation allowed by BBN. In their calculation, the sensitivity is dominated by variation of the neutron lifetime, $\tau$, which strongly affects the ${ }^{4} \mathrm{He}$ abundances which are well constrained observationally. We have shown that this calculation is based on a modeldependent assumption. Under the reasonable assumption that all elementary particle masses are proportional to $v$, the Higgs vacuum expectation value, the sensitivity of $\tau$ to $v$ is shown to be quite large (although only half that found by Ref. [28]), and we obtain a limit $|\delta v / v|<0.9 \%$, which within this model translates into $\left|\delta m_{q} / m_{q}\right|<0.9 \%$.

In Refs. [12,13] also, bounds are derived from the ${ }^{7} \mathrm{Li}$ abundance that are different from zero, namely $\delta m_{q} / m_{q}=$ $0.016 \pm 0.005$ and $\delta m_{q} / m_{q}=0.013 \pm 0.002$, respectively. Also here, the uncertainties do not include the 
theoretical uncertainty of the input quantities. In our work we have not included the ${ }^{7} \mathrm{Li}$ abundance for several reasons. Firstly, the ${ }^{7} \mathrm{Li}$ abundance is very sensitive to variations in ${ }^{7} \mathrm{Be}$ and ${ }^{7} \mathrm{Li}$ binding energies, as well as $A=5$ resonances [13], which have not yet been calculated using the methodology of this paper. In the future, our study can be extended into this regime, as soon as systematic studies of the quark mass dependence of heavier nuclei are available, e.g., employing methods of nuclear lattice calculations-see Refs. [88,89] and references therein. In fact, the first results within that framework for ${ }^{4} \mathrm{He},{ }^{8} \mathrm{Be}$ and ${ }^{12} \mathrm{C}$ can be found in Ref. [7]. Secondly, the discrepancy between theory and observation is a factor of $\sim 3$, which can make nonlinear effects important. Lastly, the observational status and interpretation of the "Spite plateau" of lithium abundances in Population III stars is still uncertain (see, e.g., Refs. $[83,90,91]$ and references therein).

\section{ACKNOWLEDGMENTS}

C. H. acknowledges the hospitality and the funding by UNSW, where a part of this work was carried out. J. N. acknowledges funding by Fundación Ramón Areces. This work is supported in part by Spanish Research Contracts No. FPA2007-29115-E/, No. FPA2011-27853-C02-02, and No. FPA2008-05287-E/FPA "Quark Masses and Hadron Physics (From Quarks to Life)," by the Australian Research Council, by the DFG and the NSFC through funds provided to the Sino-German CRC 110 "Symmetries and the Emergence of Structure in QCD," by the HGF through funds provided to the Nuclear Astrophysics Virtual Institute (NAVI; VH-VI-417), by the BMBF (06BN9006), and by the EU via ERC Project No. 259218 NuclearEFT and the EU FP7 HadronPhysics3 project.
[1] J.-P. Uzan, Rev. Mod. Phys. 75, 403 (2003); Living Rev. Relativity 14, 2 (2011).

[2] K. A. Olive, M. Pospelov, Y.-Z. Qian, A. Coc, M. Cassé, and E. Vangioni-Flam, Phys. Rev. D 66, 045022 (2002).

[3] R. L. Jaffe, A. Jenkins, and I. Kimchi, Phys. Rev. D 79, 065014 (2009).

[4] C. J. Hogan, Rev. Mod. Phys. 72, 1149 (2000).

[5] M. Livio, D. Hollowell, A. Weiss, and J. W. Truran, Nature (London) 340, 281 (1989).

[6] H. Oberhummer, A. Csoto, and H. Schlattl, Science 289, 88 (2000).

[7] E. Epelbaum, H. Krebs, T. A. Lähde, D. Lee, and U.-G. Meißner, Phys. Rev. Lett. 110, 112502 (2013).

[8] J. K. Webb, J. A. King, M. T. Murphy, V. V. Flambaum, R. F. Carswell, and M. B. Bainbridge, Phys. Rev. Lett. 107, 191101 (2011); J. A. King, J. K. Webb, M. T. Murphy, V. V. Flambaum, R. F. Carswell, M. B. Bainbridge, M. R. Wilczynska, and F. E. Koch, Mon. Not. R. Astron. Soc. 422, 3370 (2012).

[9] J. C. Berengut, V. V. Flambaum, J. A. King, S. J. Curran, and J. K. Webb, Phys. Rev. D 83, 123506 (2011).

[10] T. Dent, S. Stern, and C. Wetterich, Phys. Rev. D 76, 063513 (2007).

[11] W. J. Marciano, Phys. Rev. Lett. 52, 489 (1984); X. Calmet and H. Fritzsch, Eur. Phys. J. C 24, 639 (2002); P. Langacker, G. Segré, and M. J. Strassler, Phys. Lett. B 528, 121 (2002); C. Wetterich, J. Cosmol. Astropart. Phys. 10 (2003) 002; T. Dent and M. Fairbairn, Nucl. Phys. B653, 256 (2003).

[12] V. V. Flambaum and R. B. Wiringa, Phys. Rev. C 76, 054002 (2007).

[13] J. C. Berengut, V. V. Flambaum, and V. F. Dmitriev, Phys. Lett. B 683, 114 (2010).

[14] O. Civitarese, M. A. Moliné, and M.E. Mosquera, Nucl. Phys. A846, 157 (2010).
[15] A. Coc, P. Descouvemont, K. A. Olive, J.-P. Uzan, and E. Vangioni, Phys. Rev. D 86, 043529 (2012).

[16] V. V. Flambaum and E. V. Shuryak, Phys. Rev. D 65, 103503 (2002); 67, 083507 (2003).

[17] V. V. Flambaum, A. Holl, P. Jaikumar, C. D. Roberts, and S. V. Wright, Few-Body Syst. 38, 31 (2006).

[18] A. Holl, P. Maris, C. D. Roberts, and S. V. Wright, Nucl. Phys. B, Proc. Suppl. 161, 87 (2006).

[19] V. V. Flambaum, arXiv:physics/0309107.

[20] V. V. Flambaum, D. B. Leinweber, A. W. Thomas, and R. D. Young, Phys. Rev. D 69, 115006 (2004).

[21] M.E. Carrillo-Serrano, I. C. Cloët, K. Tsushima, A. W. Thomas, and I. R. Afnan, Phys. Rev. C 87, 015801 (2013).

[22] H. Leutwyler, Ann. Phys. (N.Y.) 235, 165 (1994).

[23] P.C. Bruns and U.-G. Meißner, Eur. Phys. J. C 40, 97 (2005).

[24] C. Hanhart, J. R. Peláez, and G. Rios, Phys. Rev. Lett. 100, 152001 (2008).

[25] J. R. Peláez and G. Rios, Phys. Rev. D 82, 114002 (2010).

[26] J. Nebreda and J. R. Peláez, Phys. Rev. D 81, 054035 (2010).

[27] J. Gasser and U.-G. Meißner, Nucl. Phys. B357, 90 (1991).

[28] P. F. Bedaque, T. Luu, and L. Platter, Phys. Rev. C 83, 045803 (2011).

[29] E. Epelbaum, U.-G. Meißner, and W. Glöckle, Nucl. Phys. A714, 535 (2003).

[30] S. R. Beane and M. J. Savage, Nucl. Phys. A713, 148 (2003).

[31] E. Epelbaum, U.-G. Meißner, W. Glöckle, and C. Elster, Phys. Rev. C 65, 044001 (2002).

[32] V. Bernard, N. Kaiser, and U.-G. Meißner, Int. J. Mod. Phys. E 04, 193 (1995); Nucl. Phys. A615, 483 (1997).

[33] J. Soto and J. Tarrus, Phys. Rev. C 85, 044001 (2012).

[34] J. Mondejar and J. Soto, Eur. Phys. J. A 32, 77 (2007).

[35] J. F. Donoghue, Phys. Rev. C 74, 024002 (2006).

[36] J. F. Donoghue, Phys. Lett. B 643, 165 (2006). 
[37] S. Dürr et al., Science 322, 1224 (2008).

[38] A. Walker-Loud, in Workshop on Chiral Dynamics, Jefferson Lab, Newport News, VA, 2012 (to be published).

[39] M. Frink, U.-G. Meißner, and I. Scheller, Eur. Phys. J. A 24, 395 (2005).

[40] M. Albaladejo and J. A. Oller, Phys. Rev. D 86, 034003 (2012).

[41] J. M. Alarcon, J. Martin Camalich, and J. A. Oller, Phys. Rev. D 85, 051503 (2012).

[42] J. Gasser, H. Leutwyler, and M.E. Sainio, Phys. Lett. B 253, 252 (1991).

[43] C. Ditsche, M. Hoferichter, B. Kubis, and U.-G. Meißner, J. High Energy Phys. 06 (2012) 043.

[44] M. Hoferichter, C. Ditsche, B. Kubis, and U.-G. Meißner, J. High Energy Phys. 06 (2012) 063.

[45] G. S. Bali et al., Nucl. Phys. B866, 1 (2013).

[46] A. S. Kronfeld, Annu. Rev. Nucl. Part. Sci. 62, 265 (2012).

[47] J. Bijnens, Prog. Part. Nucl. Phys. 58, 521 (2007).

[48] J. Gasser and H. Leutwyler, Nucl. Phys. B250, 465 (1985); B250, 517 (1985); B250, 539 (1985).

[49] G. Amoros, J. Bijnens, and P. Talavera, Nucl. Phys. B602, 87 (2001).

[50] G. Colangelo, J. Gasser, and H. Leutwyler, Nucl. Phys. B603, 125 (2001).

[51] A. Bazavov et al. (MILC Collaboration), Proc. Sci. CD 09 (2009) 007.

[52] G. Colangelo et al., Eur. Phys. J. C 71, 1695 (2011).

[53] C. W. Bernard, T. Burch, K. Orginos, D. Toussaint, T. DeGrand, C. DeTar, S. Datta, S. Gottlieb, U. Heller, and R. Sugar, Phys. Rev. D 64, 054506 (2001); Ph. Boucaud et al. (ETM Collaboration), Phys. Lett. B 650, 304 (2007); C. Allton et al. (RBC and UKQCD Collaborations), Phys. Rev. D 76, 014504 (2007).

[54] X. Feng, K. Jansen, and D. B. Renner, Phys. Rev. D 83, 094505 (2011).

[55] C. R. Allton, W. Armour, D. B. Leinweber, A. W. Thomas, and R.D. Young, Phys. Lett. B 628, 125 (2005); M. Göckeler et al. (QCDSF Collaboration), Proc. Sci. LATTICE 08 (2008) 136.

[56] T. N. Truong, Phys. Rev. Lett. 61, 2526 (1988); 67, 2260 (1991); A. Dobado, M. J. Herrero, and T. N. Truong, Phys. Lett. B 235, 134 (1990); A. Dobado and J. R. Peláez, Phys. Rev. D 47, 4883 (1993); 56, 3057 (1997).

[57] J. Nebreda, J. R. Peláez, and G. Rios, Phys. Rev. D 83, 094011 (2011).

[58] S. Aoki et al., Phys. Rev. D 60, 114508 (1999).

[59] E. Epelbaum, W. Glöckle, and U.-G. Meißner, Eur. Phys. J. A 19, 125 (2004).

[60] J. Gasser and H. Leutwyler, Ann. Phys. (N.Y.) 158, 142 (1984).

[61] N. Fettes, Ph.D. thesis, University Bonn, 2000.

[62] V. Bernard and U.-G. Meißner, Phys. Lett. B 639, 278 (2006).
[63] R. G. Edwards, G. Fleming, Ph. Hägler, J. Negele, K. Orginos, A. Pochinsky, D. Renner, D. Richards, and W. Schroers (LHPC Collaboration), Phys. Rev. Lett. 96, 052001 (2006).

[64] V. Bernard, Prog. Part. Nucl. Phys. 60, 82 (2008).

[65] E. Epelbaum, W. Glöckle, and U.-G. Meißner, Eur. Phys. J. A 19, 401 (2004).

[66] E. Epelbaum, W. Glöckle, and U.-G. Meißner, Nucl. Phys. A747, 362 (2005).

[67] R. Machleidt, K. Holinde, and Ch. Elster, Phys. Rep. 149, 1 (1987).

[68] E. Braaten and H.-W. Hammer, Phys. Rev. Lett. 91, 102002 (2003).

[69] E. Epelbaum, H.-W. Hammer, U.-G. Meißner, and A. Nogga, Eur. Phys. J. C 48, 169 (2006).

[70] E. Epelbaum, U.-G. Meißner, and W. Glöckle, arXiv:nucl-th/0208040.

[71] S. R. Beane and M.J. Savage, Nucl. Phys. A717, 91 (2003).

[72] S. R. Beane, P. F. Bedaque, K. Orginos, and M. J. Savage, Phys. Rev. Lett. 97, 012001 (2006).

[73] S. R. Beane, P.F. Bedaque, M.J. Savage, and U. van Kolck, Nucl. Phys. A700, 377 (2002).

[74] J.-W. Chen, T.-K. Lee, C.-P. Liu, and Y.-S. Liu, Phys. Rev. C 86, 054001 (2012).

[75] T. Yamazaki, K.-i. Ishikawa, Y. Kuramashi, and A. Ukawa, Phys. Rev. D 86, 074514 (2012).

[76] L. Kawano, Report No. FERMILAB-Pub-92/04-A, 1992.

[77] C. Angulo et al., Nucl. Phys. A656, 3 (1999).

[78] R. H. Cyburt, Phys. Rev. D 70, 023505 (2004).

[79] S. Ando, R. H. Cyburt, S. W. Hong, and C. H. Hyun, Phys. Rev. C 74, 025809 (2006).

[80] R.H. Cyburt and B. Davids, Phys. Rev. C 78, 064614 (2008).

[81] E. Segré, Nuclei and Particles (Benjamin-Cummings, Reading, MA, 1977).

[82] D. Larson et al., Astrophys. J. Suppl. Ser. 192, 16 (2011).

[83] J. Beringer et al. (Particle Data Group), Phys. Rev. D 86, 010001 (2012).

[84] R. H. Cyburt, B. D. Fields, and K. A. Olive, J. Cosmol. Astropart. Phys. 11 (2008) 012.

[85] J. Gasser and H. Leutwyler, Phys. Rep. 87, 77 (1982).

[86] A. Walker-Loud, C.E. Carlson, and G. A. Miller, Phys. Rev. Lett. 108, 232301 (2012).

[87] A. Coc, N. J. Nunes, K. A. Olive, J.-P. Uzan, and E. Vangioni, Phys. Rev. D 76, 023511 (2007).

[88] E. Epelbaum, H. Krebs, D. Lee, and U.-G. Meißner, Phys. Rev. Lett. 106, 192501 (2011).

[89] E. Epelbaum, H. Krebs, T. Lähde, D. Lee, and U.-G. Meißner, Phys. Rev. Lett. 109, 252501 (2012).

[90] F. Iocco and M. Pato, Phys. Rev. Lett. 109, 021102 (2012).

[91] K. A. Olive, P. Petitjean, E. Vangioni, and J. Silk, Mon. Not. R. Astron. Soc. 426, 1427 (2012). 